\title{
Evolution of Gaussian wave packets in capillary jets
}

\author{
F. J. García $\odot,{ }^{1}$ H. González $\odot,{ }^{2, *}$ F. J. Gómez-Aguilar $\odot,{ }^{1}$ A. A. Castrejón-Pita $\odot,{ }^{3}$ and J. R. Castrejón-Pita $\odot^{4}$ \\ ${ }^{1}$ Departamento de Física Aplicada I, Escuela Politécnica Superior, Universidad de Sevilla, c/ Virgen de África, 7, 41011-Sevilla, Spain \\ ${ }^{2}$ Departamento de Física Aplicada III. Escuela Técnica Superior de Ingeniería, Universidad de Sevilla, Camino de los Descubrimientos, \\ s/n, 41092-Sevilla, Spain \\ ${ }^{3}$ Department of Engineering Science, University of Oxford, Parks Road, Oxford OX1 3PJ, United Kingdom \\ ${ }^{4}$ School of Engineering and Material Science, Queen Mary University of London, Mile End Road, London E1 4NS, United Kingdom
}

(Received 17 July 2019; revised manuscript received 7 August 2019; published 26 November 2019)

\begin{abstract}
A temporal analysis of the evolution of Gaussian wave packets in cylindrical capillary jets is presented through both a linear two-mode formulation and a one-dimensional nonlinear numerical scheme. These analyses are normally applicable to arbitrary initial conditions but our study focuses on pure-impulsive ones. Linear and nonlinear findings give consistent results in the stages for which the linear theory is valid. The inverse Fourier transforms representing the formal linear solution for the jet shape is both numerically evaluated and approximated by closed formulas. After a transient, these formulas predict an almost Gaussian-shape deformation with (i) a progressive drift of the carrier wave number to that given by the maximum of the Rayleigh dispersion relation, (ii) a progressive increase of its bell width, and (iii) a quasiexponential growth of its amplitude. These parameters agree with those extracted from the fittings of Gaussian wave packets to the numerical simulations. Experimental results are also reported on near-Gaussian pulses perturbing the exit velocity of a 2-mm diameter water jet. The possibility of controlling the breakup location along the jet and other features, such as pinch-off simultaneity, are demonstrated.
\end{abstract}

DOI: 10.1103/PhysRevE.100.053111

\section{INTRODUCTION}

A liquid, when sufficiently pressurized, is allowed to escape, in the form of a jet, through an orifice to break up into a string of droplets [1]. Savart [2] in 1833 experimentally demonstrated that the liquid jets are destabilized by any disturbance in the nozzle, which, however small, grows up downstream and causes its rupture. Plateau [3] in 1873 showed that surface tension is responsible for the destabilization of any cylindrical liquid columns under the axisymmetric perturbations of a sufficiently long wavelength. Rayleigh [4,5] in 1879, in his analysis, included the balance between surface tension and inertia, and determined how these perturbations grow and the typical size of the droplets that are thus formed. Since then, there have been numerous extensions of Lord Rayleigh's perturbative analysis that have included other effects, as viscosity, gravity, surrounding atmosphere, electric and magnetic fields, thermal gradients, viscoelasticity, and compound jets $[6,7]$.

Technologically, capillary jetting is an advantageous method of droplet production. It allows the generation of drops of well-determined size (monodisperse) with a meticulous control over their position and speed. Since the invention of the first continuous ink-jet printers [8,9], the industrial applications of capillary jets have grown to include a large variety of materials, to the point where these methods are used in other fields, such as pellet manufacturing (metals, fertilizers, detergents), drug dosing, live tissue engineering, cell sorting

*helio@us.es or cell encapsulation, and encapsulation of pharmaceutical products or food, among others $[9,10]$.

From a theoretical point of view, there are two possible approaches that can be used to study the evolution of liquid jets: the temporal analysis and the spatiotemporal analysis (or simply spatial analysis). In the temporal analysis, Rayleigh [4] modelled a liquid jet as an infinite liquid column seen from a reference system that moves with the speed of the jet. The perturbations of such an infinite jet can be described as a superposition of Fourier modes in the axial coordinate, whose amplitudes change in time (initial value problem). Keller et al. [11] in 1973, noting that Rayleigh's temporal analysis is an approximation, proposed a more rigorous approach. In the so-called spatial analysis, the temporal dependency of the perturbations is described in terms of a Fourier superposition, while the perturbations grow spatially downstream subject to the exit boundary conditions in the orifice (boundary value problem). Keller himself showed that the temporal analysis coincides with the spatial one in the limit in which the Weber number We (square of the ratio between the velocity of the jet and the capillary speed) goes to infinity. In practice, the temporal analysis is simple and works very well for $\mathrm{We} \geqslant 20$ [12-15], a condition that is fulfilled in most applications based on liquid jets. Here we have adopted a temporal approach, but a spatial analysis would be necessary for slower jets [16].

Regardless of the analysis adopted, and even for axisymmetric jets, the mathematical problem to be solved remains difficult: It is a three-dimensional free boundary problem (the moving surface of the jet) with cylindrical geometry, which evolves over time and is, in general, highly nonlinear [6]. Even the linear analysis for small harmonic perturbations is 
difficult, especially in the viscous case [17], whose solution requires numerical methods [18]. The system is dispersive and unstable, and the nature of the instability changes from convective to absolute depending on the liquid and jetting properties [6]. In this context, the so-called one-dimensional (1D) models offer a very useful simplification [19-22]. The spatial dependence of the 3D equations can be reduced to the axial variable by assuming a very simplified polynomial radial dependence, which is justified by the slenderness of the liquid column $[21,23]$.

The resulting equations remain nonlinear, but the spatial dimensionality is reduced by eliminating the radial dependence. In this way, our spatial domain is fixed, in contrast to the 3D domain, which has a free boundary. The rigorous deduction of these models from the Navier-Stokes equations can determine the accuracy of each 1D model [21,23-25]. For low viscosities, the analysis of small perturbations has shown that in the range of wave numbers of $0<k<1$, where the liquid jet is unstable, the viscous slice model [21,22], which is a generalization of the nonviscous slice model of Lee [19], results in errors of up to $6 \%$ in the description of the jet's temporal evolution. The Cosserat model [20] is accurate for zero or very low viscosities but carries an error of up to $3 \%$ for moderate or large viscosities. In the present work, we use the so-called averaged model [21] with a minor correction $[14,24]$. The averaged model is the most accurate $1 \mathrm{D}$ method with a $0.6 \%$ error in the entire parametric range of interest. Moreover, its difficulty is similar, both for numerical simulations and for perturbative analysis. Its accuracy in the nonlinear regime is as good as in the linear regime, and it well describes the jet close to the breakup point [24].

In the classical analyses of harmonic perturbations $[4,17]$ only a single mode of evolution (the one responsible for instability) was selected, disregarding the contribution of other modes that can be found in the corresponding dispersion relation. These classical monomodal analyses predict that the amplitude of harmonic disturbances should grow exponentially. On the other hand, there are numerous numerical and experimental examples of initial nonexponential transients that show that a single mode is unable to couple the boundary conditions at the orifice with the subsequent evolution of the liquid jet. Recently, a rigorous modal analysis has revealed that, among the infinite modes that arise from the 3D dispersion relation, only two (but not less) are required to adequately describe the initial conditions in the temporal analysis [14] or the boundary conditions at the orifice in the spatial analysis $[16,26]$. In brief, the two retained modes are those having non-negligible shape deformation and net axial flow or, in mathematical terms, those surviving to a radial integration over any jet section. None of the neglected modes is unstable. The relevant quantity associated to the velocity field is its axial component averaged on a slice, which is related to the shape deformation by means of a mass balance, in a similar way as in 1D models [see Eq. (21) in Sec. IV]. Together with the well-known dominant (unstable) capillary mode, the subdominant (stable) capillary mode is also essential. The same conclusion is applicable to 1D models [27]. This bimodal analysis simplifies the calculations and makes them more comprehensible and, at the same time, accurately describes the early evolution of the capillary jet subjected to

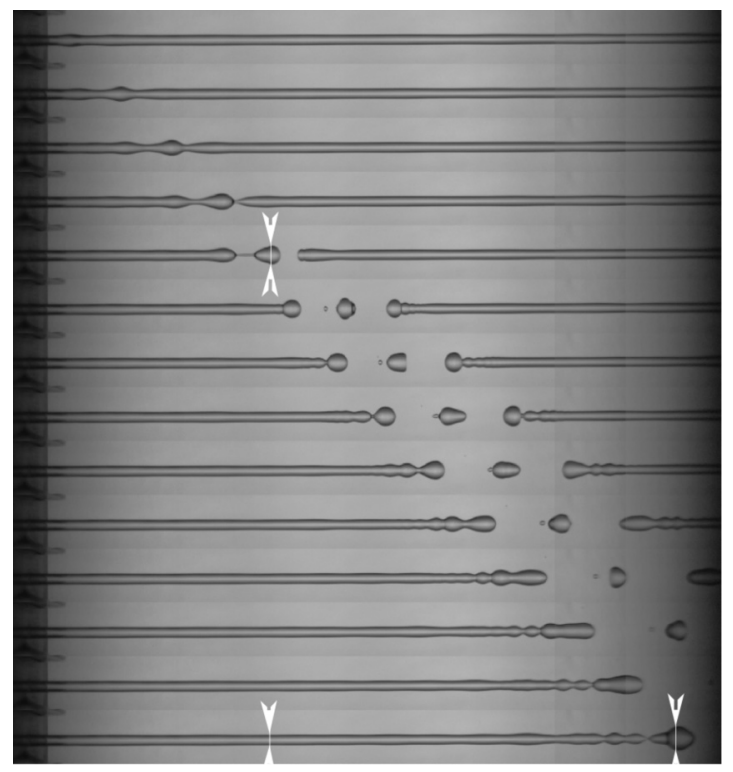

FIG. 1. Sequence of frames, for equally elapsed times, corresponding to the evolution of a capillary jet perturbed by a pressure pulse at the nozzle. The marks indicate the first and second detachment events, clearly separated in space.

arbitrary conditions in the nozzle [28]. In this work, we apply realistic initial conditions of pure impulse (without initial deformation), therefore adopting a bimodal analysis.

In the context of inkjet printing, two different technologies stand out: continuous inkjet (CIJ) and drop on demand (DOD). In CIJ, droplets are continuously generated from a truly formed jet by a periodic stimulation. During operation, only some of the droplets are electrostatically deflected for printing, while the others are recycled [8]. In contrast, in DOD, droplets are only produced when needed [10]. While CIJ printing can handle a large variety of liquids, and yields a high droplet production per unit of time and a high printing speed, DOD printing normally generates small droplets and its design facilitates scaling up so that a single printhead can hold hundreds of nozzles [9]. In this work, we study a lesser known hybrid technology, which we name the pulsed stimulation of continuous jets. In 1989, Hrdina and Crowley [29] experimentally demonstrated the possibility of producing isolated drops in a continuous stream, without appreciably disturbing the rest of the jet. In this approach, drops are generated by intermittently stimulating a small jet length by electric fields. Single drops can be produced on demand, but with the advantages of a CIJ system. Other authors have numerically studied this problem to predict the breakup length of the jet and the shape of the rupture into drops [30-33]. However, the presence of an electric field complicates the study of the evolution of pulsed perturbations in capillary jets. In particular, nozzle stimulation by pressure pulses is an approach that has not been studied in the past. We can see an example from our experiments in Fig. 1.

Concerning the shape of the stimulation pulse, there are good reasons for choosing a Gaussian wave packet. Although any pulsed stimulation can be described by a Fourier analysis, a large number of Fourier components may be 
necessary to describe it adequately. In contrast, the same pulsed stimulation is more efficiently described in terms of wave packets, defined over a restricted domain. Among all the possible forms of wave packets, the Gaussian envelope has interesting properties. It is a smooth analytical function with a simple Fourier transform (a Gaussian function). In addition, it fulfills the principle of indetermination with an equal sign, so it is the least-dispersed function in a linear system [34]. The Gaussian wave packet roughly retains its functional form as it evolves and only by widening and changing its amplitude $[35,36]$. In unstable flows with pulsed stimulation, Gaussian wave packets have been used in systems such as inclined plane films [37], boundary layers [38,39], wakes [40], and parallel flows in general $[40,41]$. Remarkably, in the context of the Orr-Somerfield equation, Gaster [40] showed that any pulse located in space and time tends to asymptotically become a Gaussian wave packet. In liquid capillary jets, wave packets are often mentioned in the context of the transition between absolute and convective instability [42]. However, the evolution of Gaussian packets has not been studied or been considered as a stimulation mechanism to produce drops.

In this paper, we study in detail the temporal evolution of an impulsive Gaussian wave packet in an infinite liquid jet. Through a rigorous small-disturbance analysis, we demonstrate that the packet, very approximately, retains its functional form with predictable width, amplitude, and wave number. To this end, we carry out three different approximations. In the first one, described in Sec. II, we formally solve the linear problem by means of a rigorous Fourier analysis, which is numerically implemented through a fast Fourier transform. Next, in Sec. III, we propose a simple analytical approach, in which the carrier wave number, width, and amplitude of a Gaussian wave packet are explicitly obtained. Finally, in the same section, a more elaborate implicit approximation is also presented. The results of Secs. II and III allow us to physically interpret the results intuitively. We validate all these linear models with nonlinear 1D numerical simulations, as described in Sec. IV. Results from linear and nonlinear models are shown and compared in Sec. V, and their significance and scope are discussed in Sec. VI. Finally, Sec. VII presents experiments where isolated drops are obtained and controlled by the stimulation of appropriate wave packets.

\section{TWO-MODE LINEAR TEMPORAL FORMULATION OF THE JET EVOLUTION}

The temporal analysis assumes a capillary jet as an infinite liquid column at rest. The physical parameters are the liquid density $\rho$, its dynamic viscosity $\mu$, and its surface tension $\gamma$. The effects of the outer gas dynamics and that of the gravity forces are disregarded. As usual, we adopt scales based on capillary forces balanced by inertia, i.e., jet radius $R$ for lengths, the capillary time $t_{c} \equiv\left(\rho R^{3} / \gamma\right)^{1 / 2}$ for time, and the capillary velocity $v_{c} \equiv R / t_{c}$ for velocities. The sole dimensionless number in this formulation is the Ohnesorge number $\mathrm{Oh} \equiv \mu /(\rho R \gamma)^{1 / 2}$. In what follows, we only deal with dimensionless quantities.

The relevant quantities to formulate the axisymmetric hydrodynamical problem are the jet deformation, $F(z, t)$, and the instantaneous mean axial velocity on a slice $W(z, t)$, which are functions of the axial position $z$ and time $t[14,16]$. A sketch is provided in Fig. 2. This is valid both for a twomode linear analysis, presented in this section, and for the one-dimensional nonlinear formulation that will serve to build a numerical scheme in Sec. IV.

A perturbed jet can be represented by

$$
F(z, t)=1+f(z, t) ; W(z, t)=w(z, t),
$$

where $f(z, t)$ and $w(z, t)$ are the small-amplitude deformation and velocity perturbations, respectively. Note that for the temporal analysis framework, the jet velocity of the unperturbed state is null. In this work, $f(z, t)$ and $w(z, t)$ have wavepacket forms for which we define their respective Fourier decompositions,

$$
f(z, t)=\frac{1}{2 \pi} \int_{-\infty}^{\infty} d k e^{i k z} \hat{f}(k, t)
$$

and

$$
w(z, t)=\frac{1}{2 \pi} \int_{-\infty}^{\infty} d k e^{i k z} \hat{w}(k, t) .
$$

Each Fourier component can be considered a harmonic perturbation, whose behavior has been analyzed in García and González [14] in terms of their initial values $\hat{f}(k, 0)$ and $\hat{w}(k, 0)$. In brief, these spectral components are the sum of two contributions, which are the evolutions of the capillary dominant, and capillary subdominant modes,

$$
\hat{f}(k, t)=\hat{f}_{d}(k, t)+\hat{f}_{s}(k, t)
$$

with

$$
\hat{f}_{d}(k, t)=\frac{-e^{\alpha_{d}(k) t}}{\alpha_{d}(k)-\alpha_{s}(k)}\left[\alpha_{s} \hat{f}(k, 0)+\frac{i k}{2} \hat{w}(k, 0)\right] .
$$

Here $\alpha_{d}(k)$ and $\alpha_{s}(k)$ are the growth rates of the dominant and subdominant modes, respectively. Although $\alpha_{d}(k)$ and $\alpha_{s}(k)$ can be obtained by numerically solving the dispersion relation corresponding to the $3 \mathrm{D}$ linear equations, in this work we use the simpler formulas given by the average model, which are accurate enough [14]. Equation (5) also defines $\hat{f}_{s}(k, t)$ by merely interchanging the subindices "d" and "s." This interchange rule holds throughout the text. Similar formulas for the velocity perturbation can be consulted in García and González [14], but they will not be necessary in this work.

Although Eq. (5) is valid for arbitrary initial conditions, we are mainly interested in pure-impulse conditions, which are suitable for rigid exit orifices, and hence we set $\hat{f}(k, 0)=0$. By combining all the spectral components in each mode we obtain

$$
f(z, t)=f_{d}(z, t)+f_{s}(z, t)
$$

with

$$
f_{d}(z, t)=\frac{-i}{2 \pi} \int_{-\infty}^{\infty} d k \exp \left[i k z+\alpha_{d}(k) t\right] g(k) \hat{w}(k, 0),
$$

where we define

$$
g(k) \equiv \frac{k / 2}{\alpha_{d}(k)-\alpha_{s}(k)},
$$

intended as a transfer function connecting any initial velocity perturbation to the initial amplitude of the capillary dominant 

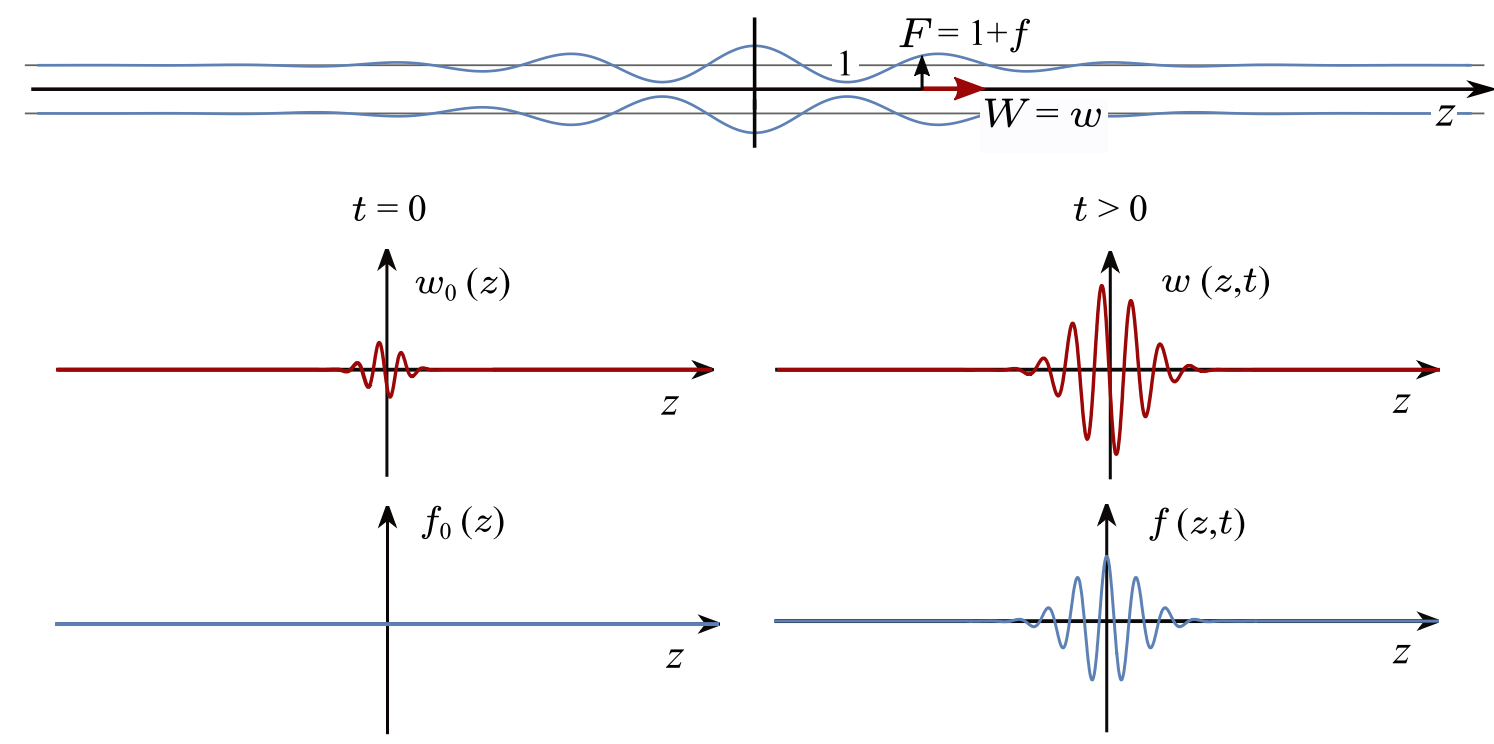

FIG. 2. Sketch of the physical system (on top) showing the quantities defined in Sec. II. The jet is considered as an infinite column at rest, with a shape deformation $F(z, t)$ and an average axial velocity (on a slice) $W(z, t)$. The relations with their perturbations, $f(z, t)$ and $w(z, t)$, are also provided. Below the system sketch, on the left, typical pure-impulse initial conditions, defined by a pulse perturbation in the averaged axial velocity, $w_{0}(z)$, and a null shape perturbation, $f_{0}(z)=0$. On the right, the same quantities at a later time $t>0$.

mode (changing the sign for the subdominant mode). Hereinafter, we use $f_{d}(z, t)$ as the dominant wave packet and $f_{s}(z, t)$ as the subdominant wave packet.

Let us consider an initial velocity perturbation given by a Gaussian wave-packet pulse:

$$
W(z, 0)=w(z, 0)=-w_{0} \exp \left(-\frac{z^{2}}{2 \sigma_{0}^{2}}\right) \sin \left(k_{0} z+\phi_{0}\right),
$$

where $w_{0}$ is its amplitude, $\sigma_{0}$ its width, $k_{0}$ its carrier wave number, and $\phi_{0}$ the initial phase shift relative to the center of the Gaussian bell. Its Fourier transform is

$$
\begin{aligned}
\hat{w}(k, 0)= & -w_{0} \int_{-\infty}^{\infty} d z e^{-i k z} \exp \left(-\frac{z^{2}}{2 \sigma^{2}}\right) \sin \left(k_{0} z+\phi_{0}\right) \\
= & \frac{i w_{0}}{\sqrt{2}} \sqrt{\pi} \sigma_{0}\left\{\exp \left[-\frac{1}{2} \sigma_{0}^{2}\left(k-k_{0}\right)^{2}+i \phi_{0}\right]\right. \\
& \left.-\exp \left[-\frac{1}{2} \sigma_{0}^{2}\left(k+k_{0}\right)^{2}-i \phi_{0}\right]\right\}
\end{aligned}
$$

The formal solution to the evolution of a initial velocity Gaussian wave packet is obtained by substituting Eq. (10) into (7), yielding

$$
\begin{aligned}
f_{d}(z, t)= & \frac{w_{0} \sigma_{0}}{2 \sqrt{2 \pi}} \int_{-\infty}^{\infty} d k g(k) \exp \left[i k z+\alpha_{d}(k) t\right] \\
& \times\left\{\exp \left[-\frac{1}{2} \sigma_{0}^{2}\left(k-k_{0}\right)^{2}+i \phi_{0}\right]\right. \\
& \left.-\exp \left[-\frac{1}{2} \sigma_{0}^{2}\left(k+k_{0}\right)^{2}-i \phi_{0}\right]\right\}
\end{aligned}
$$

$f_{s}(z, t)$ is given by a similar formula through the mentioned interchange of indices.

The inverse Fourier transform in Eq. (11) can be efficiently evaluated with the help of a FFT algorithm [43] to provide the spatial distribution of the wave packet at any time.
However, considerable insight can be obtained from the analytical approximations presented in the next section.

\section{ANALYTICAL APPROXIMATIONS}

First, we focus our attention on the evolution of the dominant wave packet. The integrand in Eq. (11) can be split into two terms, each of them having the contribution of one Gaussian bell, centered in $k_{0}$ and $-k_{0}$, respectively. Let us consider the first one. When $t$ is large enough, the integrand has an asymptotically dominant maximum at $k=k_{m}$, defined by the conditions $\alpha_{d}^{\prime}\left(k_{m}\right)=0$, where the prime denotes derivation with respect to the argument; indeed, $\alpha_{d}^{\prime \prime}\left(k_{m}\right)<0$, so $k_{m}$ corresponds to a maximum. Therefore, the structure of this integral is well suited for the application of the Laplace method [44] to obtain an asymptotic approximation as $t \rightarrow$ $\infty$. At the leading order of $1 / t$, the behavior is

$$
\begin{aligned}
\int_{-\infty}^{\infty} & d k g(k) \exp \left[i k z+\alpha_{d}(k) t-\frac{1}{2} \sigma_{0}^{2}\left(k-k_{0}\right)^{2}+i \phi_{0}\right] \\
\sim & g\left(k_{m}\right) \exp \left[\alpha_{d}\left(k_{m}\right) t-\frac{1}{2} \sigma_{0}^{2}\left(k_{m}-k_{0}\right)^{2}+i \phi_{0}\right] \\
& \times \int_{-\infty}^{\infty} d k \exp \left[i k z+\frac{1}{2} \alpha_{d}^{\prime \prime}\left(k_{m}\right)\left(k-k_{m}\right)^{2} t\right] \\
= & g\left(k_{m}\right) \exp \left[\alpha_{d}\left(k_{m}\right) t-\frac{1}{2} \sigma_{0}^{2}\left(k_{m}-k_{0}\right)^{2}\right] \\
& \times \sqrt{\frac{-2 \pi}{\alpha_{d}^{\prime \prime}\left(k_{m}\right) t}} \exp \left[\frac{z^{2}}{2 \alpha_{d}^{\prime \prime}\left(k_{m}\right) t}\right] \exp \left(i k_{m} z+i \phi_{0}\right) .
\end{aligned}
$$

The complementary Gaussian, centered at $-k_{0}$, admits the same procedure and gives a similar result. The combination of both asymptotic approximations yields a Gaussian wave packet deformation with an amplitude growing according to $\exp \left[\alpha_{d}\left(k_{m}\right) t\right] / \sqrt{t}$, a growing bell width of the form 
$\left[-\alpha_{d}^{\prime \prime}\left(k_{m}\right) t\right]^{1 / 2}$ and a carrier wave number $k_{m}$. Although these findings are interesting and connect with similar results in the literature [38], their application can be of limited use when the jet has a relatively short breakup time. Thus, we look for a nonasymptotic approximation, which is valid for finite times, based on the hypothesis of a large Gaussian bell width. In the following subsections, we propose two analytical approaches, which are showing the near-Gaussian nature of the packet throughout the most part of its evolution, with one of them giving simpler explicit formulas for the defining parameters, while the other one provides more accurate but implicit formulas.

\section{A. Explicit approximation}

As in the previous asymptotic analysis, we again consider the first term in Eq. (11) after it has been split into two integrals. First, $g(k)$ is written in exponential form, $\exp \{\ln [g(k)])\}$, in order to express the whole integrand as an exponential function whose exponent becomes $S_{d}(k)+i \phi_{0}+i k z$, where

$$
S_{d}(k) \equiv \ln [g(k)]-\frac{1}{2} \sigma_{0}^{2}\left(k-k_{0}\right)^{2}+\alpha_{d}(k) t .
$$

We now expand the functions $\alpha_{d}(k)$ and $\ln [g(k)]$ up to second order around $k_{m}$. In this way, $S_{d}$ becomes a quadratic polynomial that we can express as $-\sigma^{2}\left(k-k_{c}\right)^{2} / 2+b$, for some $\sigma$, $k_{c}$, and $b$ to be determined. These three parameters are easily calculated by identifying the coefficients of each power of $k$ in the polynomials and solving the resulting system of equations. After some manipulations we arrive at

$$
\begin{aligned}
\sigma & =\sqrt{\sigma_{0}^{2}-[\ln (g)]^{\prime \prime}\left(k_{m}\right)-\alpha_{d}^{\prime \prime}\left(k_{m}\right) t}, \\
k_{c} & =k_{m}+\left(k_{0}-k_{m}\right) \frac{\sigma_{0}^{2}}{\sigma^{2}}+\frac{[\ln (g)]^{\prime}\left(k_{m}\right)}{\sigma^{2}}, \\
b & =\frac{\sigma^{2}}{2}\left(k_{c}-k_{m}\right)^{2}-\frac{\sigma_{0}^{2}}{2}\left(k_{0}-k_{m}\right)^{2} .
\end{aligned}
$$

This new exponential expression allows an approximate evaluation of the integral (11) in closed form. The growing behavior of $\sigma$ makes this evaluation more accurate as $t$ increases. Recalling that $\alpha_{d}(k)$ and $\alpha_{s}(k)$ are even functions, Eqs. (14) hold when replacing $k_{0} \leftarrow-k_{0}, k_{m} \leftarrow-k_{m}$, and $k_{c} \leftarrow-k_{c}$ for the same $\sigma$ and $b$. These symmetry properties allows us to effortlessly calculate the other integral in (11) having $k+k_{0}$ in the exponent. By combining the two contributions, we resort to a Gaussian wave packet with the following structure:

$$
f(z, t)=A \exp \left(-\frac{z^{2}}{2 \sigma^{2}}\right) \cos \left(k_{c} z+\phi_{0}\right)
$$

where the amplitude is

$$
A=w_{0} g\left(k_{m}\right) \frac{\sigma_{0}}{\sigma} \exp \left[b+\alpha_{d}\left(k_{m}\right) t\right]
$$

Note that we have chosen a negative-sine-type carrier for the initial velocity, Eq. (9), so as to obtain a cosine-type carrier for the deformation, Eq. (15). In this way, taking $\phi_{0}=0$ leads to a symmetric breakup with a central main droplet. An illustration of a general Gaussian wave packet for the surface deformation is depicted in Fig. 3. The amplitude $A$ depends on $t$ explicitly but also implicitly through $b$ and $\sigma$. As time

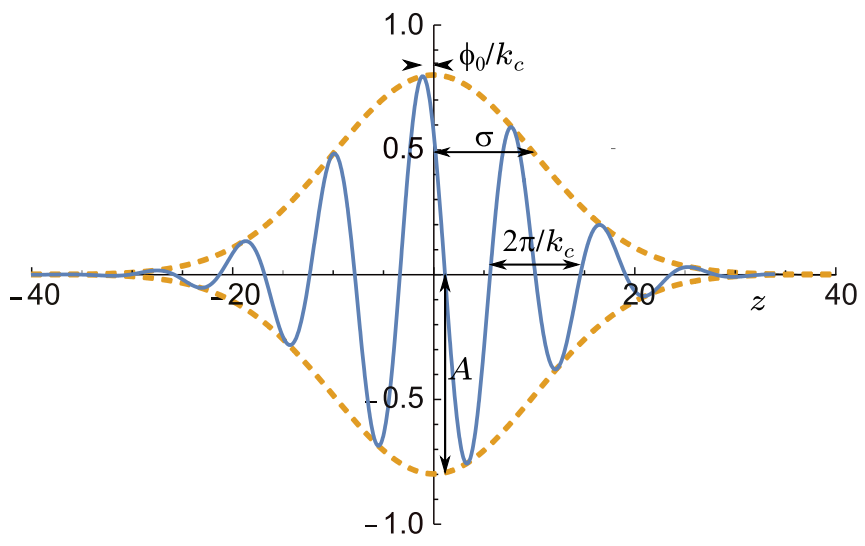

FIG. 3. A typical Gaussian wave packet as defined in Eq. (15). The dashed lines show the Gaussian envelope, characterized by its amplitude $A$ and width $\sigma$. A wavelength $2 \pi / k_{c}$ is defined for a carrier wave. A negative shift $-\phi_{0} / k_{c}$ is shown as the result of a phase $\phi_{0}$. The specific values in this illustration are $A=0.8, \sigma=10, k_{c}=0.7$, and $\phi_{0}=\pi / 4$.

elapses, the values of $k_{c}$ and $\sigma$ give the temporal evolution of the carrier wave number and the bell width, respectively. The trends are (i) an unlimited increase of the bell width that is consistent with the asymptotic prediction deduced from the Laplace method, Eq. (12); (ii) a convergence of the carrier wave number $k_{c}$ toward $k_{m}$, independently of its initial value $k_{0}$; and (iii) a quasiexponential growth of the amplitude, with the growth rate corresponding to the maximum of the Rayleigh dispersion relation, $\alpha_{d}\left(k_{m}\right)$, as $b$ tends to the constant $-\sigma_{0}^{2}\left(k_{0}-k_{m}\right)^{2} / 2$ when $t \rightarrow \infty$. As a conclusion, in this limit, Eq. (12) is recovered.

Besides these asymptotic behaviors, the proposed analytic approximation aims at reproducing, as best as possible, the whole temporal evolution of the packet. Equation (14) predicts an initial bell width and a carrier wave number that are different from $\sigma_{0}$ and $k_{0}$, respectively, because of the extra terms dependent on $g$. This surprising result will be contrasted against our numerical simulations in Sec. V and discussed in Sec. VI. Also concerning the effects of this function, it is important to note that the negative term $-[\ln (g)]^{\prime \prime}\left(k_{m}\right)$ in the expression for $\sigma$ can make the proposed explicit approximation impossible for the early stages of the temporal evolution if $\sigma_{0}$ is too small.

As a final remark, the explicit approximation can give a simple estimate of the breakup time, $t_{b}$, defined as the time required by the shape perturbation to reach an amplitude equal to the jet radius, i.e., $A\left(t_{b}\right)=1$. From this condition and Eqs. (14) and (16), we can implement the iterative procedure

$$
\begin{aligned}
\sigma_{i} & =\sqrt{\sigma_{0}^{2}-[\ln (g)]^{\prime \prime}\left(k_{m}\right)-\alpha_{d}^{\prime \prime}\left(k_{m}\right) t_{i}}, \\
k_{c, i} & =k_{m}+\left(k_{0}-k_{m}\right) \frac{\sigma_{0}^{2}}{\sigma_{i}^{2}}+\frac{[\ln (g)]^{\prime}\left(k_{m}\right)}{\sigma_{i}^{2}}, \\
b_{i} & =\frac{\sigma_{i}^{2}}{2}\left(k_{c, i}-k_{m}\right)^{2}-\frac{\sigma_{0}^{2}}{2}\left(k_{0}-k_{m}\right)^{2} . \\
t_{i+1} & =\frac{1}{\alpha_{d}\left(k_{m}\right)}\left[\ln \frac{\sigma_{i}}{\sigma_{0} w_{0} g\left(k_{m}\right)}-b_{i}\right],
\end{aligned}
$$



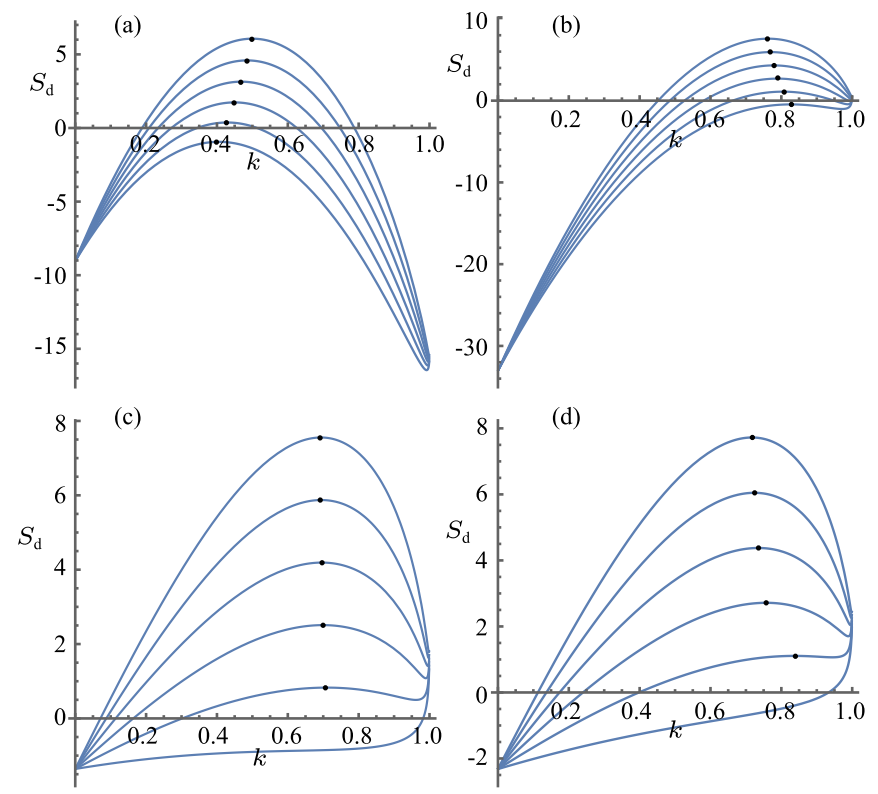

FIG. 4. The function $S_{d}(k)$, whose maximum (if existing) determines the behavior of the inverse Fourier transform in Eq. (11), is evaluated at $t=0,5,10,15$, and 20 for (a) $k_{0}=0.4, \sigma_{0}=10$; (b) $k_{0}=0.8, \sigma_{0}=10$; (c) $k_{0}=0.4, \sigma_{0}=2$; and (d) $k_{0}=0.8, \sigma_{0}=2$. In all cases, the Ohnesorge number is set to $\mathrm{Oh}=0.01$. Increasing the time makes the function increase too. The maximum of each curve is marked by a dot.

starting with $t_{1}=-\ln \left[w_{0} g\left(k_{m}\right)\right] / \alpha_{d}\left(k_{m}\right)$. The wanted estimate of the breakup time is $t_{b}=\lim _{i \rightarrow \infty} t_{i}$. Convergence within three decimal figures is typically achieved in five iterations.

\section{B. Implicit approximation}

Alternatively, a more accurate, although implicit, approximation can be envisaged by revisiting the exponent $S_{d}(k)$, Eq. (13). The first term, $\ln [g(k)]$, increases monotonically in the range of interest $0<k<1$. The second term has a maximum at $k=k_{0}$ and its relative importance depends on $\sigma_{0}^{2}$. The third term has a maximum at $k=k_{m}$ and is proportional to $t$. An analysis of $S_{d}(k)$ for different times reveals a progressive displacement of the relative maximum toward $k=k_{m}$ (see Fig. 4). The idea is to search for a better approximation of $S_{d}(k)$ by determining the position of this moving maximum and performing a Taylor expansion around it. Note that the explicit method works with the best approximation around the Rayleigh maximum, which is a region not containing the main contribution to the integral. Let us redefine $k_{c}$, dependent on $t$, as the position of this maximum (if existing), thus satisfying $S^{\prime}\left(k_{c}\right)=0$, i.e.,

$$
\sigma_{0}^{2}\left(k_{c}-k_{0}\right)-\alpha_{d}^{\prime}\left(k_{c}\right) t-[\ln (g)]^{\prime}\left(k_{c}\right)=0 .
$$

Once $k_{c}$ is found, we approximate $S_{d}(k)$ by a Taylor expansion around this value up to the second order, $S_{d}(k) \simeq S_{d}\left(k_{c}\right)+$ $S_{d}^{\prime \prime}\left(k_{c}\right)\left(k-k_{c}\right)^{2} / 2$. We also redefine

$$
\sigma \equiv \sqrt{-S_{d}^{\prime \prime}\left(k_{c}\right)}=\sqrt{\sigma_{0}^{2}-[\ln (g)]^{\prime \prime}\left(k_{c}\right)-\alpha_{d}^{\prime \prime}\left(k_{c}\right) t},
$$

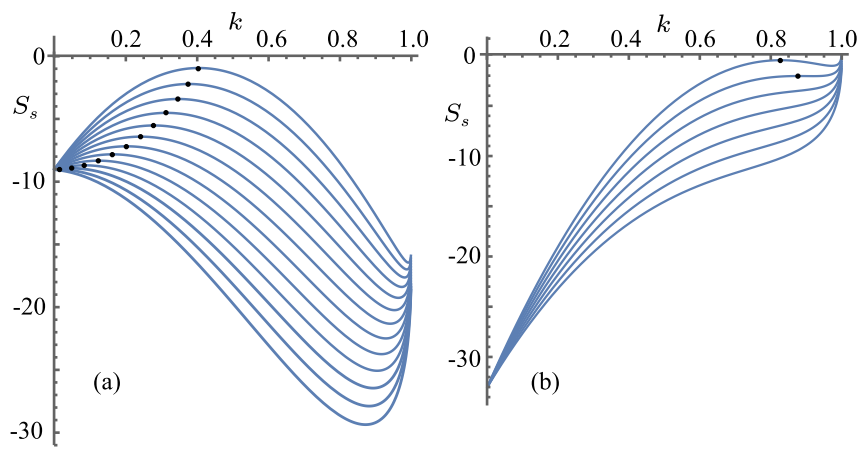

FIG. 5. (a) The function $S_{s}(k)$ for $k_{0}=0.4$ and $\sigma_{0}=10$, evaluated in the interval $t=0, \ldots, 60$ with increments of 5 in dimensionless units; (b) same evaluations for $k_{0}=0.8, \sigma_{0}=10$ and the interval $t=0, \ldots, 30$. In both cases, $\mathrm{Oh}=0.01$. Increasing the time makes the function decrease.

again dependent on $t$. The adoption of this nomenclature is fully justified below.

The new form of the exponent again allows an approximate evaluation of the integral (11) in closed form. As in the explicit approximation, and because $\alpha_{d}(k)$ and $\alpha_{s}(k)$ are even functions, Eqs. (18) and (8) predict that $-k_{c}$ is the solution when replacing $k_{0} \leftarrow-k_{0}$, while $\sigma$ is unchanged according to Eq. (19). These symmetry properties allow us to calculate the second integral in (11). By combining the two contributions, the dominant packet is again of Gaussian type and is analogous to that expressed in Eq. (15), with redefined $k_{c}, \sigma$ and $A$, in which the latter is expressed as

$$
A=w_{0} g\left(k_{c}\right) \frac{\sigma_{0}}{\sigma} \exp \left[-\frac{1}{2} \sigma_{0}^{2}\left(k_{c}-k_{0}\right)^{2}+\alpha_{d}\left(k_{c}\right) t\right] .
$$

As $t \rightarrow \infty$, the asymptotic trends of the three redefined parameters hold: from Eq. (18) it is clear that in the limit $t \rightarrow \infty$, the only possible finite solution is $\alpha_{d}^{\prime}\left(k_{c}\right)=0$, i.e., $k_{c}=k_{m}$, regardless of what the starting carrier wave number $k_{0}$ or the bell width $\sigma_{0}$ are. This fact also makes the asymptotic behavior of $\sigma$ consistent with the prediction from the Laplace method, $\left[-\alpha_{d}^{\prime \prime}\left(k_{m}\right) t\right]^{1 / 2}$. Beyond these correct trends, a better accuracy of the implicit approximation is expected because the Taylor expansion of $S_{d}(k)$ has been carried out in the neighborhood of its true maximum, i.e., the marked points in Fig. 4. This figure also shows that, as the bell width increases, the range of wave numbers providing a non-negligible contribution to the Fourier integral gets narrower and the approximation is more justified.

To complete the analysis, we consider the evolution of the subdominant wave packet, given by Eq. (11), with subindexes "d" and "s" interchanged. If we substitute $\alpha_{d}$ by $\alpha_{s}$ in (13), then we obtain the analogous function $S_{s}$, which is describing the exponent in the integral corresponding to the subdominant packet. As $\alpha_{s}(k)$ is now negative in the range of interest $0<k<1, S_{s}$ diminishes with time at each wave number but near $k=0$ (and also near $k=1$ in the inviscid case). Figure 5 illustrates the typical behavior of $S_{s}(k)$ for one initial wave number below $k_{m}$ and another beyond it. We observe that (i) for the case with $k_{0}>k_{m}, k_{c}$ does not exist after a short time, and (ii) for the case with $k_{0}<k_{m}$, the associated bell width 
diminishes and $k_{c}$ reaches the value zero at a finite time. This invalidates the application of the procedure described in this subsection and in the previous subsection to the subdominant wave packet for most of the jet evolution. Instead, in Sec. V, we show the evolution of the subdominant packet based on the numerical calculation of the inverse Fourier transform. In any case, the asymptotic trend of this packet is to decrease in amplitude, so there is a relatively extended initial transient after which the only observable packet is the dominant one.

\section{ONE-DIMENSIONAL MODEL AND NUMERICAL METHOD}

For our numerical simulations of the temporal evolution of a liquid jet (infinite jet problem), we implement the averaged one-dimensional model [21] with the minor correction introduced in García and González [14] to guarantee that its corresponding dissipation function is strictly positive. It can be written as a system of two coupled equations in terms of the already introduced surface radius $F(z, t)$ and the mean axial velocity on a slice $W(z, t)$ :

$$
\begin{gathered}
\left(F^{2}\right)_{t}+\left(F^{2} W\right)_{z}=0, \\
F^{2}\left(W_{t}+W W_{z}\right)-\left[\frac{F^{4}}{8}\left(W_{t z}-\frac{W_{z}^{2}}{2}+W W_{z z}\right)\right]_{z} \\
=-F^{2} P_{c z}+3 \mathrm{Oh}\left\{F^{2}\left[\left(1+\frac{F F_{z z}-3 F_{z}^{2}}{8}\right)^{2}+\frac{F_{z}^{4}}{2}\right] W_{z}\right\}_{z}
\end{gathered}
$$

where the subscripts $t$ and $z$ denote partial derivatives with respect to time and axial position, respectively, and $P_{c}$ is the capillary pressure jump across the surface,

$$
P_{c}=\frac{1}{\sqrt{1+F_{z}^{2}}}\left(\frac{1}{F}-\frac{F_{z z}}{1+F_{z}^{2}}\right) .
$$

Periodic boundary conditions are applied, with a domain width large enough (typically 100 times the radius of the jet) so that the evolution of the pulsed jet is the same as if the domain was infinite. Homogeneous Dirichlet boundary conditions would give identical results. Realistic initial conditions are impulsive: The liquid column is initially undeformed and the initial perturbation of the mean velocity is a Gaussian wave packet, i.e., $F(z, 0)=1$, while $W(z, 0)$ is given by Eq. (9).

The numerical method to solve the above equations is described in detail in García and González [14]. In brief, the space domain is discretized through a Galerkin finite-element method with cubic Hermite interpolation. A predictor-corrector method with variable time step is chosen to solve the resulting system of ordinary differential equations with a fixed per-step relative temporal error of $10^{-4}$. The spatial grid is refined when needed in order to guarantee a fixed per-step relative spatial error of $10^{-3}$ (up to 32000 elements typically). The results (breakup length and surface shape at breakup) do not change appreciably when reducing these error levels. The accuracy of our 1D numerical simulations is evident from the results presented in Fig. 6,

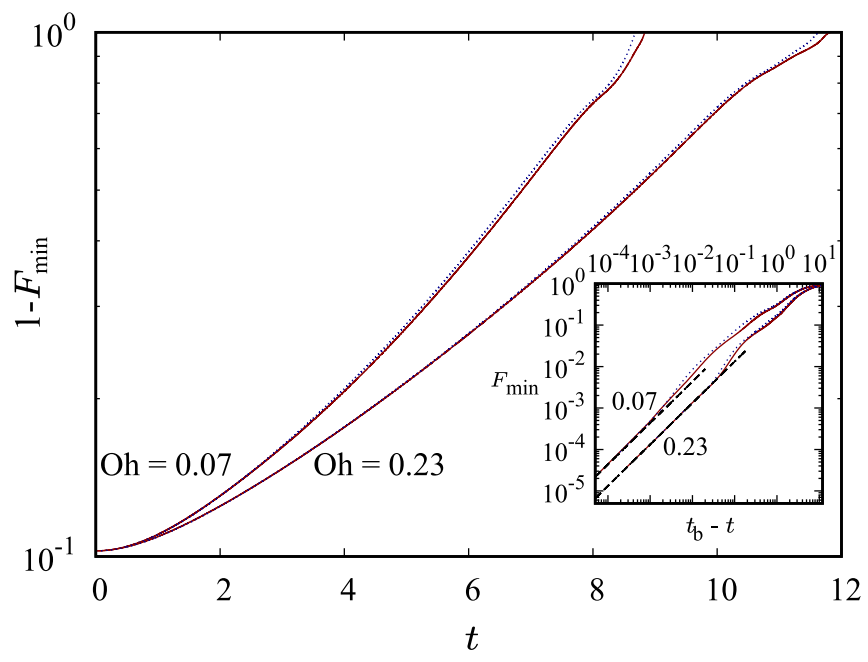

FIG. 6. Temporal evolution of the deformation amplitude of an axisymmetric capillary jet subjected to a sinusoidal perturbation of wavelength $\lambda=8$ and initial neck radius $F_{\min }=0.8975$ for $\mathrm{Oh}=$ 0.07 and 0.23 . Solid lines come from our 1D simulations, while dotted lines correspond to 3D simulations from Ref. [45]. In the inset, the neck radius is plotted in terms of the time to breakup, with the dashed line being the Eggers asymptotic solution $F_{\min }=$ $0.0304 /\left[\mathrm{Oh}\left(t-t_{b}\right)\right][46]$ near breakup.

where we contrast the ability of the model to predict the jet thinning against a well-tested 3D numerical code [45]. The same plethora of transitions described therein are reproduced here. In particular, the Eggers asymptotic solution [46] is clearly satisfied at times near breakup.

\section{RESULTS}

According to Eq. (6), the linear theory predicts a temporal evolution of the wave packet constructed as the superposition of a dominant and a subdominant wave packet. Each of them has its own evolution. The first grows in amplitude while the second decays, and both of them almost exponentially. As it happens for an harmonic stimulation [14], it is expected that, under conditions to be discussed later, the subdominant wave packet becomes negligible after an initial transient. The dominant packet has a Gaussian-like form, revealed by any of the two obtained analytical expressions. The Gaussian approximation is determined by its carrier wave number $k_{c}$, bell width $\sigma$, and amplitude $A$; all of them functions of time. In order to illustrate typical outputs from the methods implemented in this work, we present in Fig. 7 the evolution of the jet shape originated by a Gaussian-wave-packet velocity perturbation at $t=0$. The initially narrow pulse progressively widens and grows in amplitude, while its typical distance between maxima (i.e., its carrier wave number) changes. These behaviors have been predicted by our Gaussian approximations. In fact, each graph in this figure compares the implicit approximation with the $1 \mathrm{D}$ numerical findings and the numerical evaluation of the inverse Fourier transform (11), except for the first graph $(t=0.2)$, for which the analytical method cannot be evaluated (the bell width results to be an imaginary number), as warned in Sec. III. Both numerical outputs agree until the jet is close to the breakup time. The analytical output is only evaluable 

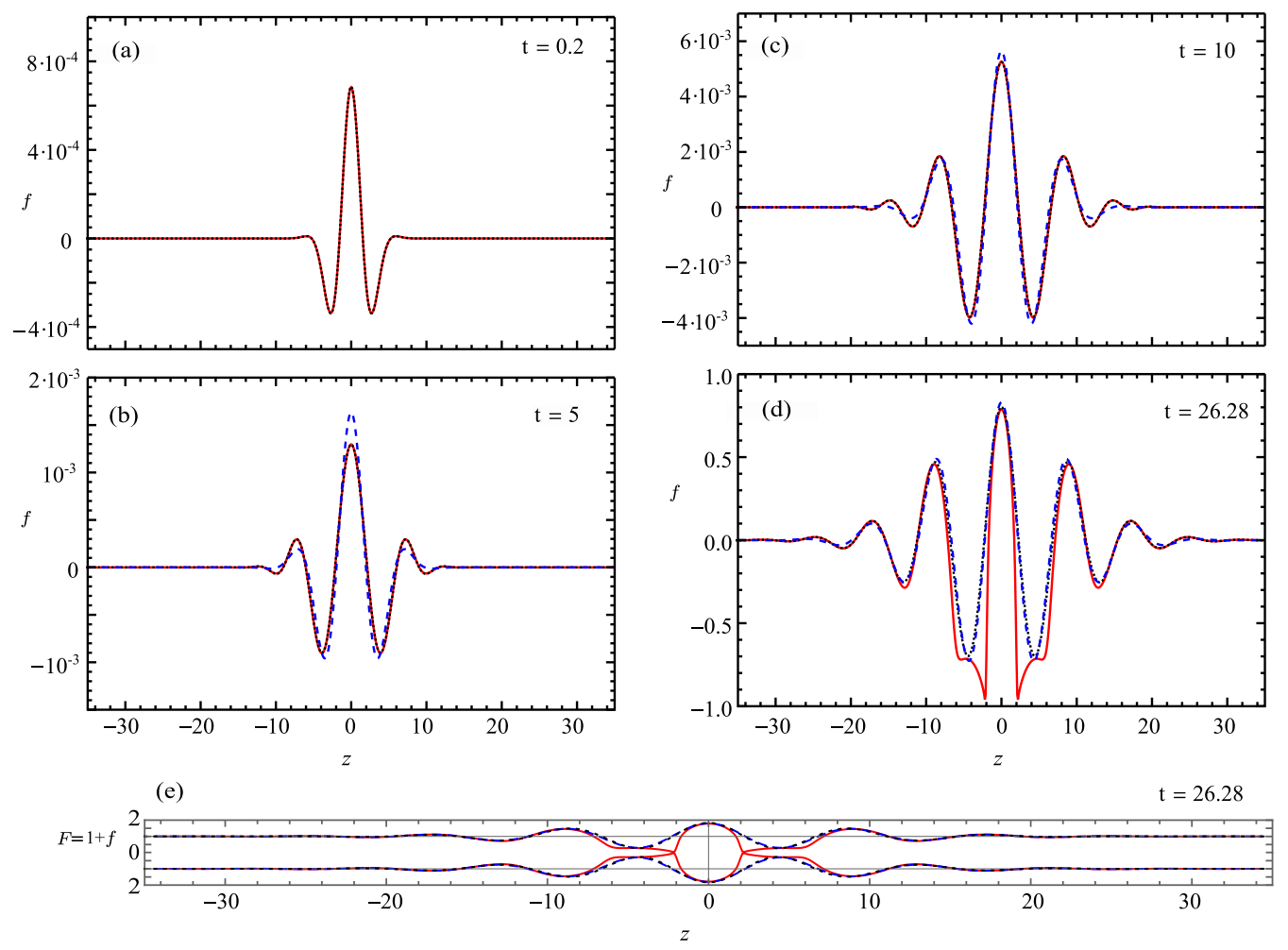

FIG. 7. The sequence (a)-(d) describes the evolution of the shape perturbation of an infinite jet, from the initial moments to the imminent breakup, for $\mathrm{Oh}=0.01$. Here, the initial jet velocity perturbation is a Gaussian wave packet with $w_{0}=0.001, k_{0}=0.69$, and $\sigma_{0}=2$. The solid lines correspond to the numerical 1D simulations; the dashed line is the numerical evaluation of the inverse Fourier transform (11); and the dotted line is the implicit approximation [absent in (a) as it is not evaluable]. Graph (d) is redrawn in full width in (e) to show $F=1+f$ (instead of $f$ ) with equal horizontal and vertical scales.

after $t=5$, and the computed inverse Fourier transform gets closer to the 1D simulation as time progresses.

The temporal evolution predicted by the numerical simulation can be analyzed by adjusting, at each time, a Gaussian wave packet, defined by the three mentioned parameters. The numerical evaluation of the inverse Fourier transform (11) admits the same fitting procedure. Accordingly, we present a comparison between the two approximate linear predictions, and the two fittings (nonlinear and exact linear) for these three parameters, in a series of figures. For instance, Fig. 8 shows the results for a packet with an initial carrier wave number $k_{c}=0.69$, corresponding to the maximum of the Rayleigh dispersion relation for $\mathrm{Oh}=0.01$ and two representative values of the initial bell width, $\sigma_{0}=10$ (wide) and $\sigma_{0}=2$ (narrow, the same as in Fig. 7). The initial amplitude is $w_{0}=$ 0.001 in all cases. In addition to the graphs for $k_{c}, \sigma$, and $A$, we plot a fourth graph with the parameter $1-r^{2}(r$, the Pearson correlation coefficient), in logarithmic scale, as a function of time, which measures how well the nonlinear simulation and the exact inverse Fourier transform for the linear problem are represented by a Gaussian wave packet. This information is important to elucidate whether eventual discrepancies in the three previous graphs come from linear transients or from nonlinearities.

The series of graphs is completed with representative values of the initial parameters: Figure 9 shows the same study as Fig. 8, this time for a fixed initial bell width $\left(\sigma_{0}=10\right)$ and different wave numbers. The cases of $k_{c}=0.6$ and 0.8 represent initial wave numbers lower and larger than $k_{m}$, correspondingly.

In order to discuss the role of the subdominant packet in the transient regime, Fig. 10 shows a measure of its temporal evolution through the maximum of the absolute value of the deformation, $f_{s, \max }(t) \equiv \operatorname{Max}_{z}\left|f_{s}(z, t)\right|$. In this semilogarithmic representation, the straight line marks an exponential decay with rate $\alpha_{s}\left(\mathrm{Oh}, k_{0}\right)$, with the same selected values of $\mathrm{Oh}$ and $k_{0}$. The decay of the packet follows this trend up to a certain saturation, which is of marginal interest. The two insets show how this saturation corresponds to Fourier components very close to $k=1$, which evolve slowly according to Fig. 5(b).

We complete the study by presenting analogous results for $\mathrm{Oh}=0.25$, which is a typical value for jets having moderate viscous effects, as those found in inkjet technology. In Fig. 11, the initial carrier wave number is fixed to the wave number of the fastest-growing perturbation for the selected Ohnesorge number, i.e., $k_{0}=k_{m}=0.57$, and shows the evolution of each parameter for a wide $\left(\sigma_{0}=10\right)$ and a narrow $\left(\sigma_{0}=2\right)$ initial bells. Figure 12 shows the comparison of a fixed initial bell width with $\sigma_{0}=10$ and two other initial carrier wave numbers, which are lower and greater than $k_{m}$, correspondingly.

\section{DISCUSSION}

Before analyzing the results presented in the previous section, we want to emphasize how the adoption of the minimal formulation of the linear problem, consisting in a combination 

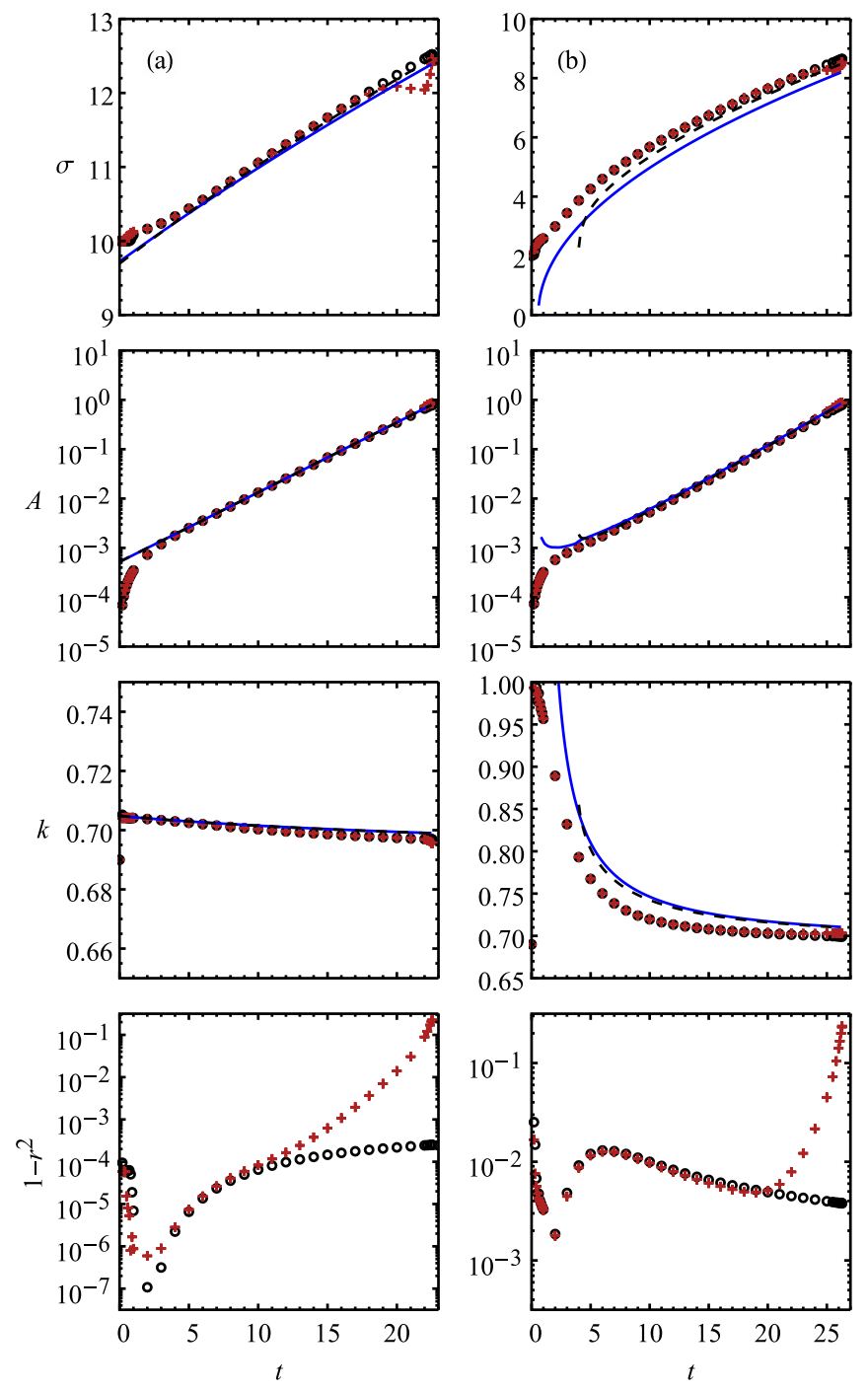

FIG. 8. For each column, the first three graphs give the bell width $\sigma$, the amplitude $A$, and the carrier wave number $k_{c}$, describing the time evolution of a wave packet generated at $t=0$ by a Gaussian wave packet perturbation of the axial jet velocity with amplitude $w_{0}=0.001$, carrier wave number $k_{0}=0.69$, and bell widths (a) $\sigma_{0}=10$ and (b) $\sigma_{0}=2$. The Ohnesorge number is $\mathrm{Oh}=0.01$ in both cases. The crosses are the values of these parameters obtained by fitting a Gaussian to the numerical simulation of the shape evolution; the circles come from a similar fitting to the numerical inverse Fourier transform; the solid lines are the values obtained from the explicit approximation of the formal solution to the linear problem; and the dashed lines are the result of the implicit approximation. The fourth graph in each column provides, in semilogarithmic scale, the quality of the fitting, measured through $1-r^{2}$, with $r$ the Pearson correlation coefficient, for the two fittings to a Gaussian wave packet.

of the two capillary modes, leads to a simple formal solution. This procedure avoids the complexity of a full modal description and captures the essential features of the physical system. The combination of a Fourier decomposition of any spatial wave packet, and the modal reduction for each $k$ component, gives much more insight than the method described in Ref. [47], based on a Laplace transform formulation of
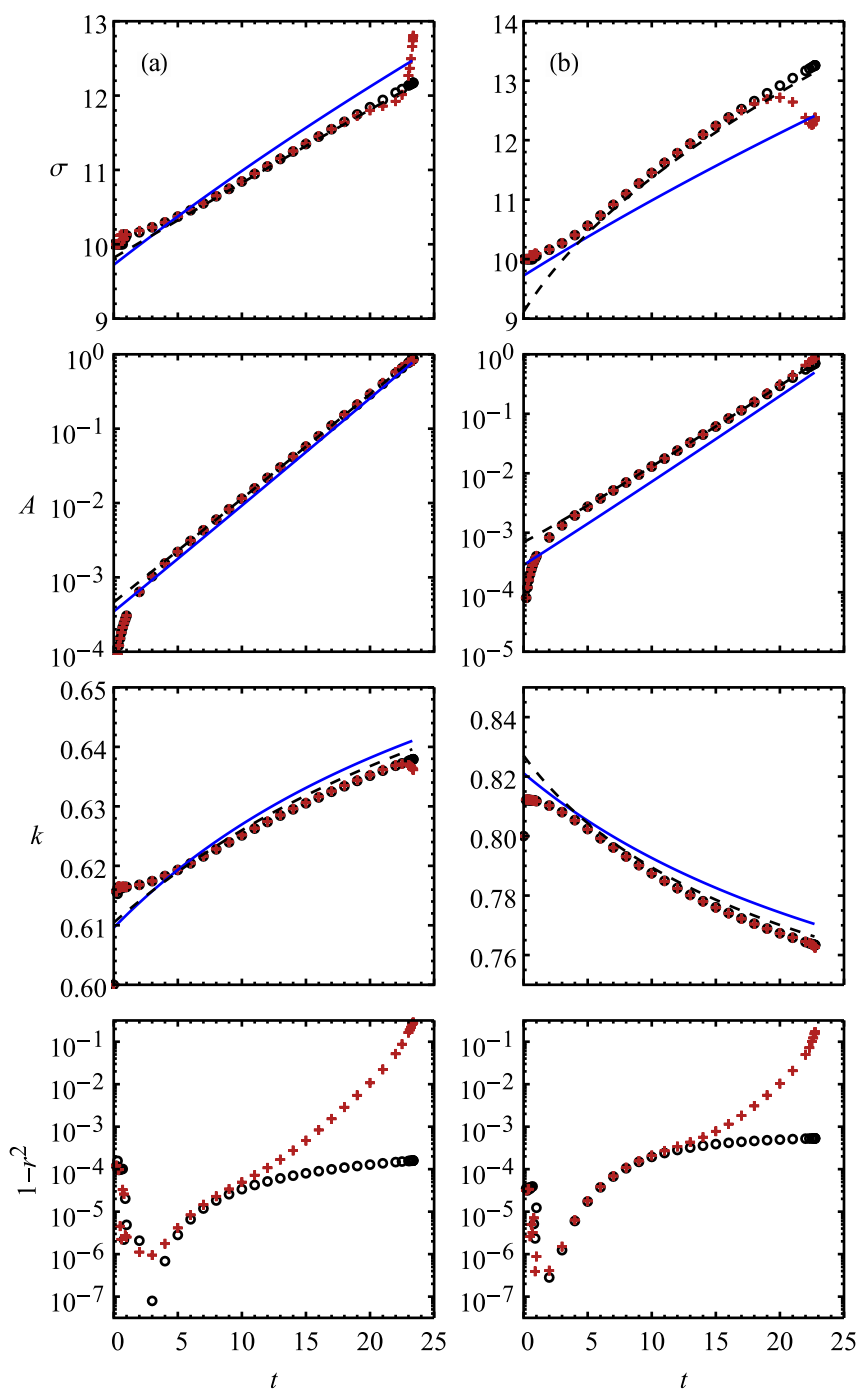

FIG. 9. Same as in Fig. 8 but for $\sigma_{0}=10$ and (a) $k_{0}=0.60$ and (b) $k_{0}=0.80$.

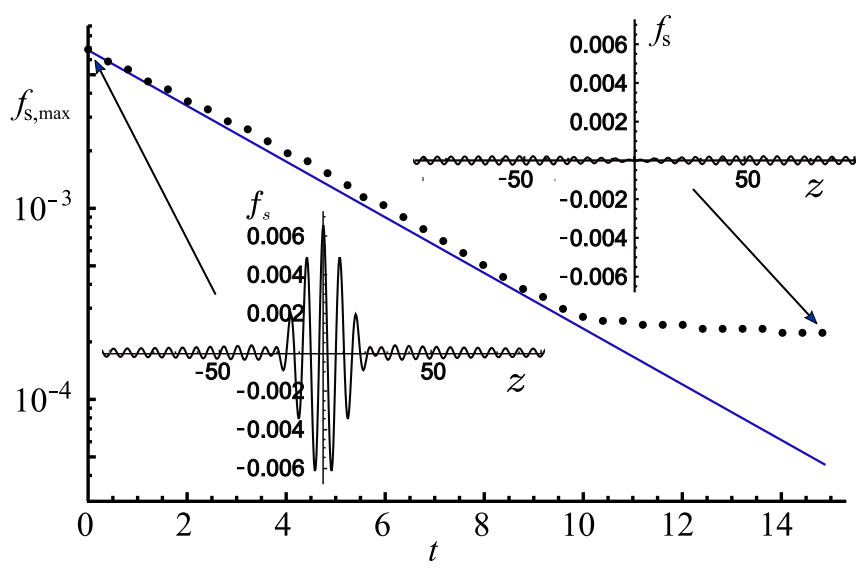

FIG. 10. Evolution of the maximum absolute value of the subdominant wave packet, $f_{\mathrm{s}, \max }(t)$, for $\mathrm{Oh}=0.01, k_{0}=0.8$, and $\sigma_{0}=$ 10. The line serves as a reference of an exponential decay with rate given by $\alpha_{s}$ for the selected values of the Ohnesorge number and the initial wave number. The insets show the wave-packet shapes corresponding to $t=0$ and $t=15$. 

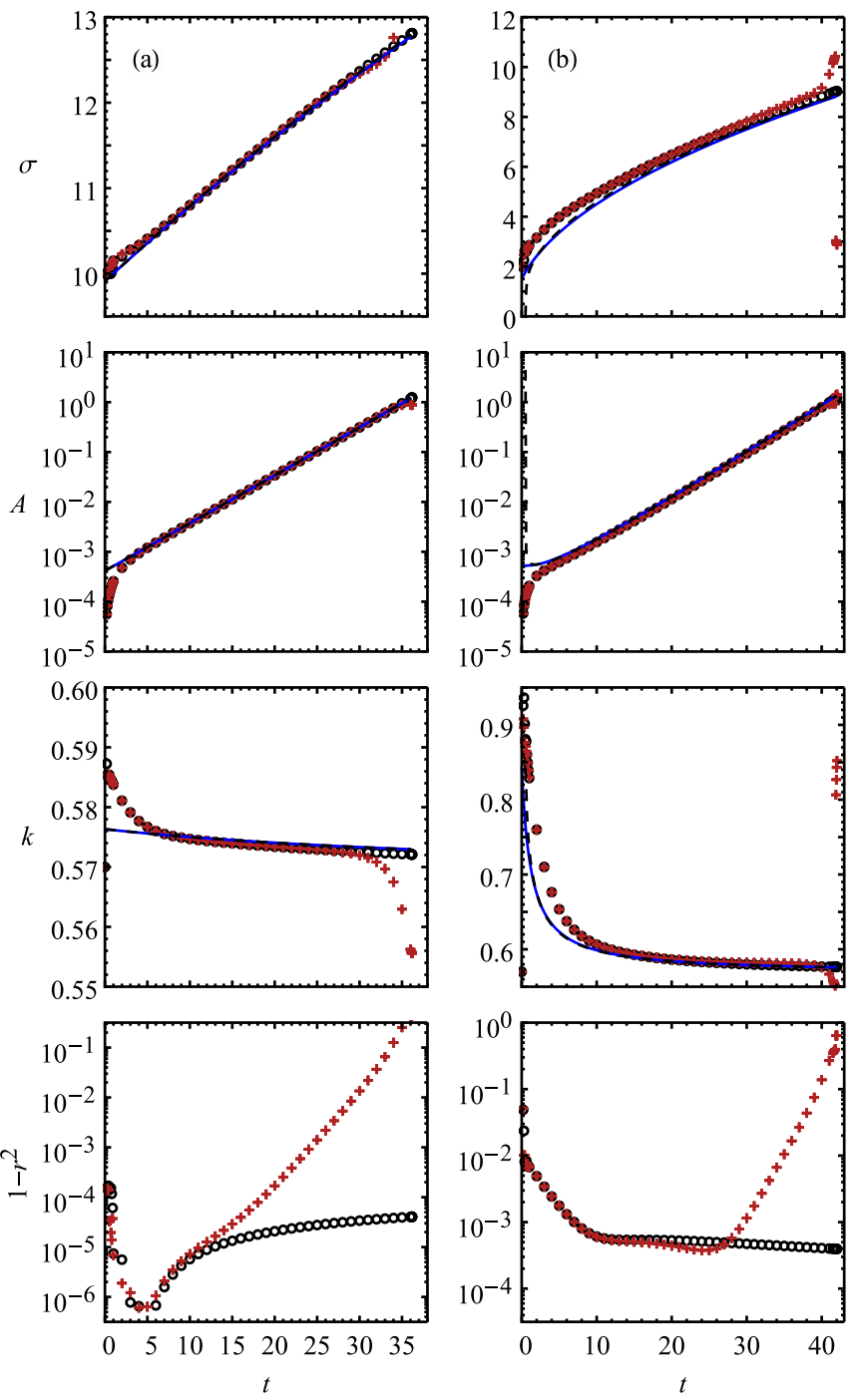

FIG. 11. Same as in Fig. 8 but for $\mathrm{Oh}=0.25$ and an initial wave number $k_{0}=0.57$ (corresponding to the maximum of the Rayleigh dispersion relation) with (a) $\sigma_{0}=10$ and (b) $\sigma_{0}=2$.

the general initial-value problem. The accuracy of our linear formulation is demonstrated in Fig. 7, where we can observe full agreement with 1D nonlinear simulations, except for the last graph, close to the breakup.

From this formal solution, the temporal evolution of a jet shape initially perturbed by a velocity wave packet can be well understood as the superposed evolution of what we have called dominant and subdominant packets. At $t=0$, the superposition gives a null deformation (pure impulsive initial condition), which starts to grow in such a way that the analytical approximations to the dominant packet mimics it very well until the final stages of the evolution, as illustrated also in Fig. 7. The temporal evolution of the amplitudes of the fitted Gaussian wave packets, compared in Figs. 8 and 9, systematically shows a transient in which the packet cannot be described by the Gaussian approximations proposed in Sec. III for the dominant packet. As the findings from the nonlinear scheme and from the numerical evaluation of the full linear solution agree during the transients, the conclusion is that the discrepancies arise from having neglected the
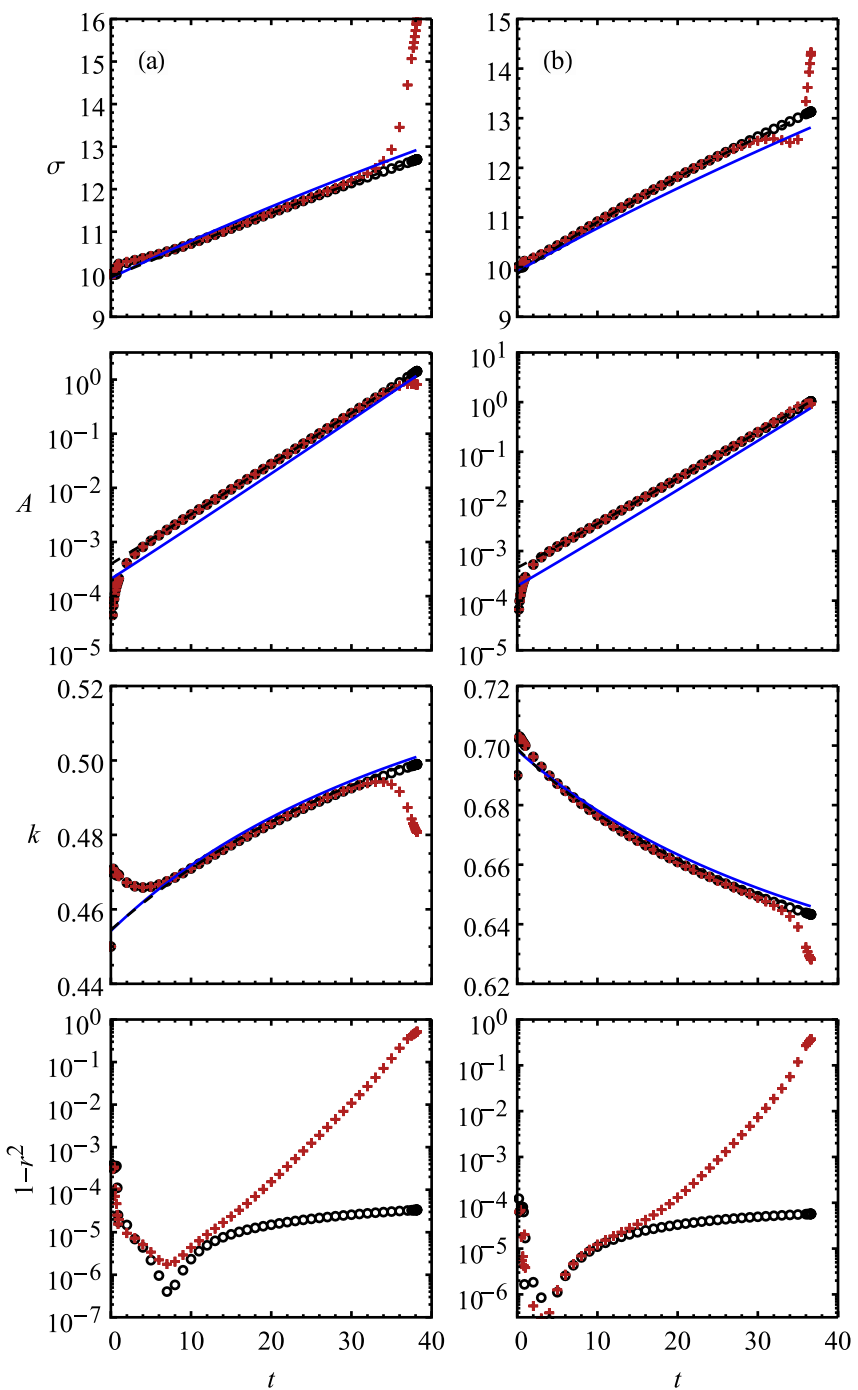

FIG. 12. Same as in Fig. 9 but for $\mathrm{Oh}=0.25$ with (a) $k_{0}=0.45$ and (b) $k_{0}=0.69$. These initial wave numbers are significantly below and above the one giving the maximum of the Rayleigh dispersion relation, $k_{0}=0.57$ [see Fig. 11(a)].

subdominant-packet contribution. The end of the transient is marked by the time beyond which there is agreement of amplitudes with those of the Gaussian approximations of the dominant packet. The transient duration will be discussed later in this section.

Concerning the performance of the linear model in describing the packet evolution, the same figures show a virtual superposition of points obtained from this linear model and from the nonlinear simulations in a wide temporal interval. As expected, discrepancies become significant only near breakup, when the amplitude cannot be considered small any more. This is clearly correlated with a worse fitting of the nonlinear predictions to the Gaussian approximation (observe the final values of the parameter $1-r^{2}$ in these figures). Once again, and similarly to cases with harmonic stimulations [14,15,28], the linear model is successful in describing most part of the temporal evolution of a perturbation in a capillary jet.

The analytical Gaussian approximations, which are proposed to represent the dominant wave packet, are successful, 
as it is apparent in Figs. 8 and 9, especially in predicting the evolution of the amplitude. In this regard, the estimates of the breakup time provided by the iterative scheme of Eq. (17) are in good agreement with the ones from the 1D nonlinear numerical computation. For instance, the evolution shown in Fig. 7 indicates a breakup time $t_{b}=26.28$ from the numerical scheme, while the iterative scheme yields $t_{b}=26.76$, i.e., a deviation lesser than $2 \%$, despite being based on a linear analysis. The other parameters are, in general, also well captured, particularly by the implicit procedure, as defined in Eqs. (18) and (19). In contrast, the explicit procedure gives a less precise agreement with the numerical points, especially for initially narrow Gaussian wave packets (see, for instance, the results for $\sigma_{0}=2$ ). The implicit approximation becomes more accurate for higher values of $\sigma_{0}$, as illustrated in Fig. 8 for the same initial wave numbers and $\sigma_{0}=10$ and $\sigma_{0}=2$. The reason is that wide bells in the spatial domain imply narrow ones in the spectral domain. Accordingly, as the only relevant contribution to the inverse transform, Eq. (11), comes from a narrow range of $k$, the remaining non-Gaussian functions in the integrand are almost constant, thus resulting in an almost Gaussian wave packet.

The persistence of a Gaussian wave-packet shape during the evolution of the jet goes beyond a mere asymptotic trend, which is not always accessible, as the jet may break up sooner. Rather, this packet shape reveals as an accurate description of the linear evolution for finite times. The three parameters describing the Gaussian approximation (disregarding the unchanging phase shift $\phi_{0}$ ), namely the amplitude, the bell width, and the carrier wave number, vary with time due to the dispersive nature of the system. Accordingly, we must aim at relating these temporal variations with the Rayleigh dispersion relation. The progressive shift of the wave number toward the maximum of the Rayleigh dispersion relation, whatever its initial value, can be viewed as a dispersive effect by which the Fourier components with higher growth rates in the wave packet progressively overtake those with lower ones. Figure 4 is very illustrative of this effect.

A particularly unexpected dispersive effect is the initial shift of the carrier wave number from its initial value $k_{0}$ to a higher one. This is due to the initial conditions considered, which are associated to velocity and not to deformations, which implies the presence of the $k$-dependent transfer function $g$. In fact, the wave packet associated to the velocity as a function of time (not included in Sec. II) does not exhibit this wave-number shift. Analogously, initial pure-deformation conditions do not produce a wave-number shift in the deformation wave packet, but in the velocity wave packet.

Recalling the analysis of the transient for harmonic perturbation, as developed by García and González [14], where explicit formulas were provided for the transient duration, the question about the possibility of similar results for packet perturbations arise. We have seen in the previous section that the subdominant packet does not have the same Gaussian behavior as the dominant one. However, Fig. 10 shows that a subdominant packet with $\sigma_{0}=10$ exhibits a decay well described by the subdominant growth rate corresponding to the selected initial carrier wave number, $k_{0}=0.8$, up to a saturation in amplitude associated to a small spectral component close to $k=1$. In fact, this component is negligible because it has a small amplitude. Even more, its presence is a spurious effect, as the dominant packet must include the same component with opposite amplitude in order to accomplish the condition of zero deformation at $t=0$ through a destructive interference of both packets. When describing the dominant packet, this tiny effect gets masked by the growth of other spectral components. As a conclusion, the application of the same explicit formulas developed in García and González [14] can give a close estimation of the transient duration, at least for the packets that are initially not too narrow.

Finally, the analysis of the results for a significantly greater viscosity $(\mathrm{Oh}=0.25)$ leads to the same conclusions about the accuracy of the linear models: The numerical inversion of the Fourier transform is virtually indistinguishable from the 1D simulations during the linear part of the evolution, and the analytical approximations give good results, especially the implicit procedure. This initial transient, along which the subdominant and dominant wave packets are of the same order of magnitude, is shorter when compared to the breakup time than for $\mathrm{Oh}=0.01$. This is consistent with the general trend presented in García and González [14] for the analysis of harmonic perturbations.

\section{PRELIMINARY EXPERIMENTS}

The previous sections constitute a self-consistent part of this article in the sense that the linear theory (along with its analytical approximations) and the nonlinear simulations give coherent results that imply mutual validation. Of course, it would be desirable to confront these theoretical results against experiments, and the goal of this research will ultimately be this one. However, this goal is difficult because (i) it may be technically very involved to impose initial Gaussian velocity perturbations, as these perturbations cannot be directly controlled, and (ii) close-up observation of the wave packet should be combined with a global visualization of the jet, in order to capture the temporal evolution of the relevant parameters (amplitude, carrier frequency, bell width, and phase). Circumventing these difficulties would require, on the one hand, mastering the whole mechanical stimulation process and, on the other hand, designing visualization strategies not currently available in the literature. For these reasons, the preliminary experiments presented in this section constitute only a first step in the experimental validation task, as well as a source of qualitative observations of very practical interest.

\section{A. Experimental setup and procedures}

The experimental set-up is described in detail elsewhere [28], so here we only give a brief outline. The jet was generated by means of a hydraulic circuit connecting in series an open reservoir receiving the falling jet, a pump, a sealed chamber that was half-filled with air (buffer), a second chamber, with one end consisting of a flexible rubber membrane connected to a mechanical shaker (LDS V201), and a cylindrical chamber (200 $\mathrm{mm}$ long, $50 \mathrm{~mm}$ in diameter), with a perforated disk to eliminate entry vortices, and a circular nozzle made from brass at the bottom $(D=2.289 \pm$ $\left.0.003 \mathrm{~mm}, \quad l_{o}=100 \pm 5 \mu \mathrm{m}\right)$. Two pressure sensors 


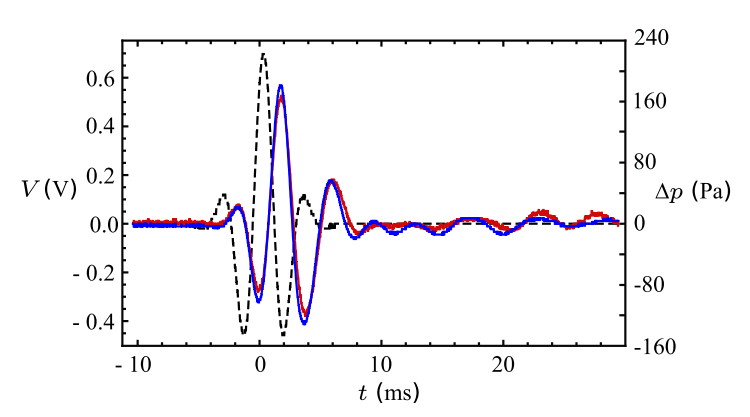

FIG. 13. Voltage packet driving the shaker (dashed line) and two independent pressure signals induced near the nozzle, as viewed on the oscilloscope screen. Scales for voltage and pressure are shown. The voltage packet corresponds to a Gaussian envelope with $0.7 \mathrm{~V}$ in amplitude and $7.15 \mathrm{~ms}$ in width; its carrier wave has a frequency of $280 \mathrm{~Hz}$ and zero phase delay.

(Honeywell 40PC006G and MEAS) were located on opposed points at the side of the cylindrical chamber, which were $12 \mathrm{~mm}$ away from the base of the nozzle plate. The jet was visualized by means of a high-speed camera (Phantom Miro 310). An acquisition rate of $48000 \mathrm{fps}$ and exposure time of $5 \mu$ s were used for all the experiments. All experiments were carried out with water $\left(\mu=1 \mathrm{mPa} \mathrm{m}^{-1}, \gamma=0.072 \mathrm{Nm}\right.$, $\left.\rho=997.6 \mathrm{~kg} / \mathrm{m}^{3}\right)$. The velocity of the jet was measured with the help of the camera software by following the first detached drop between 108 and $116 \mathrm{~mm}$ from the exit, giving $2.414 \mathrm{~m} / \mathrm{s}$. From the flow rate, fixed to $7.35 \times 10^{-6} \mathrm{~m}^{3} / \mathrm{s}$, and the measured velocity, we derive a jet radius of $0.984 \mathrm{~mm}$. These values can be adopted as valid for the whole jet length because gravity has a small effect.

The electromechanical shaker is driven by a voltage signal of Gaussian wave-packet type, periodically generated by a LabView routine. The code allows us to fix the amplitude and width of the Gaussian bell and the frequency and phase of the carrier signal, as well as the repetition rate at which this Gaussian wave packet is generated. The voltage signal is sent through a DAC board (National Instruments) to an amplifier and monitored by an oscilloscope before feeding the shaker. An example of a voltage signal used to stimulate the jet is shown in Fig. 13, just as it was viewed on the oscilloscope screen. In the same screen there were two more signals corresponding to the outputs of both pressure sensors, which are also shown in the figure, to prove the consistency of the two independent pressure perturbation measurements, $\Delta p$. The distortion between voltage and pressure signals illustrates the above mentioned difficulty in controlling the actual jet velocity perturbation. The voltage signal is proportional to the force exerted by the shaker, but the reservoir and nozzle characteristics determine the resulting pressure and flow rate temporal dependence in an uncontrolled way. From a practical point of view, we have focused our effort on (i) minimizing, by means of a proper mechanical design, the observable queue of unwanted pressure oscillations after the wave packet, and (ii) heuristically selecting the Gaussian parameters, through observation of the pressure outputs, in order to achieve a specific type of breakup (isolated, simultaneous pinch-off, etc.), as described in the next subsection.

\section{B. Applications}

The main application of pulsed stimulation is the detachment of a single drop or a group of drops in the middle of the part of the jet not affected by natural breakup. According to the previous theoretical and numerical analysis, it is expected that a packet generated by a temporal pulse will spatially grow in a quasiexponential manner, while it gets deformed much more slowly (eventual change of bell width, carrier frequency, and phase). Thus, an adequate selection of the wave-packet parameters can control the position and number of the resulting drops, as well as other relevant properties of the breakup process. This possibility is illustrated in Fig. 1, where we observe the spatiotemporal evolution of a packet generated by a Gaussian voltage pulse sent to the shaker. Specifically, conditions were designed to achieve a single-drop detachment far from a second breakup event (both marked with arrows in the figure). Note that a Gaussian wave-packet voltage pulse may not lead in general to a Gaussian-shaped deformation, as the shaker, reservoir, and nozzle dynamics mediate the velocity exit conditions. However, as the pressure perturbation measured at the reservoir only differs slightly from a Gaussian wave-packet form, we expect the same for the jet velocity perturbation at the exit.

Isolated drops, or trains of drops, can be periodically generated by periodically emitted wave-packet pulses. In this application, a question about interference between neighboring pulses naturally arises. Our analytical description of the Gaussian-pulse evolution can give a simple estimate of the minimum distance, $z_{\min }$, between two of these pulses in order to avoid unwanted interferences. The criterion to obtain this distance requires defining a maximum tolerated overlapping between two successive Gaussian envelopes, evaluated at the final stages of their evolution. We measure this overlapping through a new parameter $s$, defined as the fraction between the envelope of the pulse evaluated at $z_{\min } / 2$ (midposition between pulses) and the amplitude of the envelope, both of them at the breakup time, $t_{b}$. In this way, $z_{\min }$ is obtained through the condition

$$
s A\left(t_{b}\right)=A\left(t_{b}\right) \exp -\frac{\left(z_{\min } / 2\right)^{2}}{2 \sigma\left(t_{b}\right)^{2}},
$$

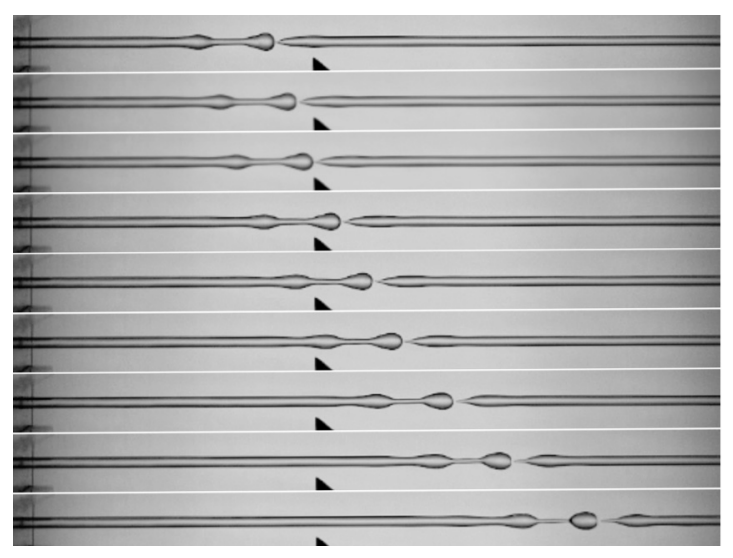

FIG. 14. Breakup events in jets stimulated by a Gaussian wavepacket pulse only differing in their initial amplitude (from $1.0 \mathrm{~V}$ to $0.2 \mathrm{~V}$ in decrements of $0.1 \mathrm{~V}$ ). The front and rear pinchings become almost simultaneous in the longest jet. 


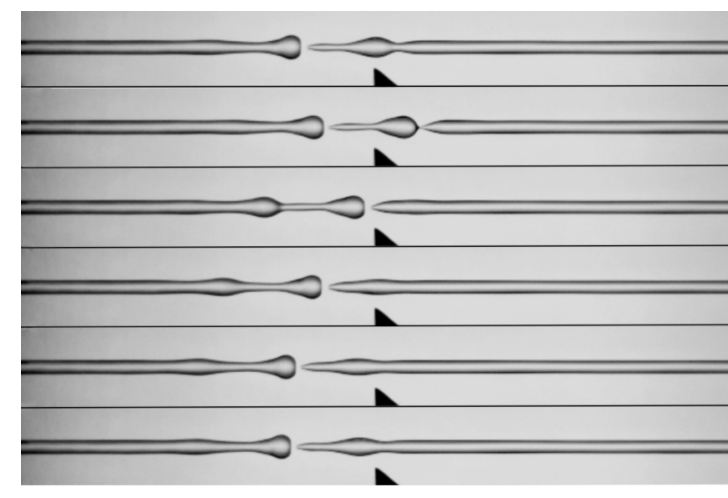

FIG. 15. Breakup events in jets stimulated by a Gaussian wavepacket pulse only differing in their phase (from $-150^{\circ}$ to $150^{\circ}$ in increments of $60^{\circ}$ ). The second frame (phase of $-90^{\circ}$ ) shows almost simultaneous pinching.

or

$$
z_{\min }=\sigma\left(t_{b}\right) \sqrt{-8 \ln s}
$$

Note that, in practice, each interfering pulse has evolved during a different lapse of time, but adopting $t_{b}$ for both of them will never underestimate $z_{\min }$. With this approximation, this distance is obtained in three steps: (i) the breakup time $t_{b}$ and its corresponding bell width, $\sigma\left(t_{b}\right)$, are estimated through the iterative scheme of Eq. (17); (ii) a prescribed tolerated overlapping parameter value $s$ is chosen, and (iii) Eq. (25) is applied to estimate the minimum distance between pulses. For instance, for the case presented in Fig. 7, the bell width at breakup is $\sigma\left(t_{b}\right) \simeq 8$.3. If we accept a total overlapping of $10 \%$, then $s=0.05$. Consequently, from Eq. (25) we obtain $z_{\min } \simeq 40.6$, which is the required separation between pulses in units of jet radius.

Recalling the unstable wave number range in the Rayleigh dispersion relation, we can note that the purely growing or decaying behavior of the capillary modes implies a coincidence between the phase velocity and the group velocity: Any packet suffers deformations but travels downstream at the same velocity than the mean flow. This is a feature that practitioners exploit to measure the flow velocity by tracking the position of a drop resulting from the isolated breakup of a conveniently tailored wave packet $[15,28]$. In this respect, it is important to achieve simultaneous pinching at the front and rear parts of the detached drop in order to minimize any unbalanced impulse. Otherwise, the drop velocity can significantly differ from that of the jet. We have observed a trend to simultaneous pinching as the breakup length increases. Figure 14 shows this effect through a comparison between several jets at their breakup instant, for different amplitudes of the initial perturbation. Independently from the origin of this behavior, probably related to the nonlinear part of the evolution, we can adjust the phase of the temporal Gaussian pulse to get simultaneous pinching. Figure 15 illustrates this possibility by a series of breakup events differing only in the phase of the stimulation. As we increase the phase, we can observe the transition from a front pinching to a rear pinching. In this way, we have the required control to produce a simultaneous pinching, no matter how long the breakup length is.

\section{CONCLUSIONS}

The linear temporal evolution of a capillary jet perturbed by a velocity pulse can be formally described as a sum of a dominant and a subdominant wave packet. The dominant wave packet is the Fourier sum of the spectral contributions of the capillary dominant mode corresponding to all the wave numbers included in the velocity pulse. The subdominant wave packet, constructed in an analogous way, decays in amplitude to an unobservable level. That moment defines the end of a transient, dependent on the Ohnesorge number, after which the linear evolution of the jet is fully described by the growth of the dominant wave packet. The duration of the transient can be estimated by resorting to the transient of a purely harmonic perturbation with the wave number equal to the carrier wave number. The final stage of the jet evolution deviates from the linear predictions when the perturbation becomes too large, as expected. Although the formulation is completely general, the described evolution has been analyzed for a specific pulse, of Gaussian type, by means of both an FFT numerical approximation of the Fourier sums and a one dimensional nonlinear simulation. Their comparison give consistent results in the linear part of the evolution. Moreover, analytical approximations of the linear formal solution predict a dominant packet still well approximated by a Gaussian with time-dependent parameters: the carrier wave number approaching that of the maximum of the Rayleigh dispersion relation, increasing bell width, and almost exponentially increasing amplitude. All these behaviors are compatible with the asymptotic trends. These analytic approximations provide a clear, simple and sufficiently accurate description of the evolution of Gaussian wave packets in capillary jets.

Preliminary experiments on pulsed stimulation demonstrate the ability to generate isolated drops in the middle of the otherwise intact length of the jet, which are well apart from the natural breakup region. Moreover, the proper adjustment of the parameters defining the Gaussian pulse allows to control key features of the breakup process for applications.

\section{ACKNOWLEDGMENTS}

We are grateful to Prof. Pierre Atten and Professor Antonio Castellanos for helpful discussions. This work was supported by the Spanish Government under Contract No. FIS2014-54539P, by the Junta de Andalucía under Contract No. P11-FQM-7919, the UK-EPSRC Starting Grant No. $\mathrm{EP} / \mathrm{P} 024173 / 1$, and an Equipment Award from the Department of Engineering Science, Oxford. A.A.C.-P. was further supported by a the Royal Society through a University Research Fellowship URF\R\180016 and an Enhancement Grant No. RGFIEA\181002

\section{APPENDIX: SOME USEFUL 1D EXPRESSIONS FOR THE EXPLICIT APPROXIMATION}

The growth rates $\alpha_{d}$ and $\alpha_{s}$ are roots of the exact 3D Rayleigh dispersion relation [48], but excellent 1D 
approximations in the whole unstable range (below a $0.5 \%$ deviation in all cases) can be obtained from the linearization of the averaged model [21], whose dispersion relation is the quadratic equation

$$
\left(1+\frac{k^{2}}{8}\right) \alpha^{2}+3 \mathrm{Oh} k^{2} \alpha-\frac{1}{2} k^{2}\left(1-k^{2}\right)=0 .
$$

By implicit differentiation of this equation and the condition $\alpha_{d}^{\prime}\left(k_{m}\right)=0$ we arrive at

$$
3 \mathrm{Oh} k_{m}^{2} \alpha_{d}\left(k_{m}\right)=-\frac{1}{8} k_{m}^{2} \alpha_{d}\left(k_{m}\right)^{2}+\frac{1}{2} k_{m}^{2}-k_{m}^{4} .
$$

In (A1), particularized for $k=k_{m}$, the substitution of its second term by (A2) and simplification leads to

$$
\alpha_{d}\left(k_{m}\right)=\frac{k_{m}^{2}}{\sqrt{2}}=2\left[4(\sqrt{2}+3 \mathrm{Oh})^{2}+1\right]^{1 / 2}-4(\sqrt{2}+3 \mathrm{Oh})
$$

From the first equality in (A3) and the formula giving the sum of the two roots,

$$
\alpha_{d}(k)+\alpha_{s}(k)=-3 \mathrm{Oh} \frac{k^{2}}{1+k^{2} / 8},
$$

at $k=k_{m}$, we obtain, again with the help of (A2),

$$
\alpha_{s}\left(k_{m}\right)=\frac{4 \sqrt{2}\left(k_{m}^{2}-1\right)}{8+k_{m}^{2}} \text {. }
$$

We also need to evaluate

$$
[\ln (g)]^{\prime}(k)=\frac{1}{k}-\frac{\alpha_{d}^{\prime}(k)-\alpha_{s}^{\prime}(k)}{\alpha_{d}(k)-\alpha_{s}(k)}
$$

and

$$
[\ln (g)]^{\prime \prime}(k)=-\frac{1}{k^{2}}-\frac{\alpha_{d}^{\prime \prime}(k)-\alpha_{s}^{\prime \prime}(k)}{\alpha_{d}(k)-\alpha_{s}(k)}+\frac{\left[\alpha_{d}^{\prime}(k)-\alpha_{s}^{\prime}(k)\right]^{2}}{\left[\alpha_{d}(k)-\alpha_{s}(k)\right]^{2}},
$$

both at $k=k_{m}$. Successive differentiations of (A4) relate the required derivatives of both roots, so we only need $\alpha_{d}^{\prime \prime}\left(k_{m}\right)$, obtained through a second implicit differentiation of (A1) to yield

$$
\alpha_{d}^{\prime \prime}\left(k_{m}\right)=-\frac{32 \sqrt{2} k_{m}^{2}}{8+k_{m}^{4}}
$$

Another helpful result is

$$
\alpha_{d}\left(k_{m}\right)-\alpha_{s}\left(k_{m}\right)=\frac{8+k_{m}^{4}}{\sqrt{2}\left(8+k_{m}^{2}\right)} .
$$

[1] E. Mariotte, Traité du Mouvement des Eaux et des Autres Corps Fluides (Michallet, E., Paris, 1686).

[2] F. Savart, Mémoire sur la constitution des veines liquides lancées par des orifices circulaires en mince paroi, Ann. Chim. 53, 337 (1833).

[3] J. A. F. Plateau, Statique Expérimentale et Théorique des Liquides Soumis aux Seules Forces Moléculaires, Vol. 2 (Gauthier Villars, Paris, 1873).

[4] L. Rayleigh, On the instability of jets, Proc. Lond. Math. Soc. 10, 4 (1878).

[5] L. Rayleigh, On the capillary phenomena of jets, Proc. R. Soc. London 29, 71 (1879).

[6] J. Eggers, Nonlinear dynamics and breakup of free-surface flows, Rev. Mod. Phys. 69, 865 (1997).

[7] J. Eggers and E. Villermaux, Physics of liquid jets, Rep. Prog. Phys. 71, 036601 (2008).

[8] R. G. Sweet, High frequency recording with electrostatically deflected ink jets, Rev. Sci. Instrum. 36, 131 (1965).

[9] S. Hoath, Fundamentals of Inkjet Printing: The Science of Inkjet and Droplets (Wiley, New York, 2015).

[10] O. A. Basaran, Small-scale free surface flows with breakup: Drop formation and emerging applications, AIChE J. 48, 1842 (2002).

[11] J. B. Keller, S. I. Rubinow, and Y. O. Tu, Spatial instability of a jet, Phys. Fluids 16, 2052 (1973).

[12] A. Kalaaji, B. Lopez, P. Attané, and A. Soucemarianadin, Breakup length of forced liquid jets, Phys. Fluids 15, 2469 (2003).

[13] H. González and F. J. García, Comment on "Breakup length of forced liquid jets" [Phys. Fluids 15, 2469 (2003)], Phys. Fluids 18, 019101 (2006).
[14] F. J. García and H. González, Normal-mode linear analysis and initial conditions of capillary jets, J. Fluid Mech. 602, 81 (2008).

[15] H. González and F. J. García, The measurement of growth rates in capillary jets, J. Fluid Mech. 619, 179 (2009).

[16] H. González, P. A. Vazquez, F. J. García, and J. Guerrero, Minimal formulation of the linear spatial analysis of capillary jets: Validity of the two-mode approach, Phys. Rev. Fluids 3, 044802 (2018).

[17] L. Rayleigh, On the instability of a cylinder of viscous liquid under capillary forces, Philos. Mag. 34, 145 (1882).

[18] S. Chandrasekhar, Hydrodynamic and Hydromagnetic Stability (Oxford University, New York/London, 1981) [Reprinted 1981, Dover, New York, 1961].

[19] H. C. Lee, Drop formation in a liquid jet, IBM J. Res. Develop. 18, 364 (1974).

[20] A. E. Green, On the nonlinear behavior of fluid jets, Int. J. Eng. Sci. 14, 49 (1976).

[21] F. J. García and A. Castellanos, One-dimensional models for slender axysimmetric viscous liquid jets, Phys. Fluids 6, 2676 (1994).

[22] J. Eggers and T. F. Dupont, Drop formation in a onedimensional aproximation of the Navier-Stokes equations, J. Fluid Mech. 262, 202 (1994).

[23] F. J. García and A. Castellanos, One-dimensional models for slender axisymmetric viscous liquid bridges, Phys. Fluids 8, 2837 (1996).

[24] F. J. García and A. Castellanos, 3-D and 1-D dynamics of slender liquid jets: Linear analysis with electric field and accuracy of 1-D models near the breakup, in Proceedings of the 1999 Conference on Electrical Insulation and Dielectric Phenomena (IEEE, Austin, TX, USA, 1999), pp. 346-349. 
[25] H. J. Subramani, H. K. Yeoh, R. Suryo, Q. Xu, B. Ambravaneswaran, and O. A. Basaran, Simplicity and complexity in a dripping faucet, Phys. Fluids 18, 032106 (2006).

[26] J. Guerrero, H. González, and F. J. García, Spatial modes of capillary jets, with application to surface stimulation, J. Fluid Mech. 702, 354 (2012).

[27] J. Guerrero, H. González, and F. J. García, Spatial modes in onedimensional models for capillary jets, Phys. Rev. E 93, 033102 (2016).

[28] F. J. García, H. González, J. R. Castrejón-Pita, and A. A. Castrejón-Pita, The breakup length of harmonically stimulated capillary jets, Appl. Phys. Lett. 105, 094104 (2014).

[29] D. W. Hrdina and J. M. Crowley, Drop-on-demand operation of continuous jets using EHD techniques, IEEE Trans. Ind. Appl. 25, 705 (1989).

[30] C. Bardeau, D. Fressard, P. Atten, and B. Barbet, Formation of isolated drops in a continuous jet, in Proceedings of the $I S \& T$ 10th Int. Congress Adv. Non-Impact Printing Tech. (Society for Imaging Science, New Orleans, Louisiana, USA, 1994), pp. 429-433.

[31] P. Atten, D. Fressard, B. Barbet, and C. Bardeau, Electrohydrodynamic induction of isolated drops in a jet, in Proceedings of the 9th International Conference on Electrostatics (Institute of Physics Conf. Ser. 143, York, 1995), pp. 47-50.

[32] B. Barbet, Stimulations electrohydrodynamique et thermique de jets de liquide conducteur, Ph.D. thesis, Université Joseph Fourier-Grenoble 1 (1997).

[33] F. J. García, Aplicación de modelos unidimensionales a la dinámica de columnas líquidas con y sin campo eléctrico, Ph.D. thesis, Universidad de Sevilla, Seville, Spain (1998).

[34] A. Papoulis, The Fourier Integral and Its Applications (McGraw-Hill, New York, 1962).

[35] C. Coulson and A. Jeffrey, Waves: A Mathematical Approach to the Common Types of Wave Motion (Longman, New York, 1977).
[36] K. Mita, Dispersion of non-gaussian free particle wave packets, Am. J. Phys. 75, 950 (2007).

[37] T. B. Benjamin, The development of three-dimensional disturbances in an unstable film of liquid flowing down an inclined plane, J. Fluid Mech. 10, 401 (1961).

[38] M. Gaster, The development of three-dimensional wave packets in a boundary layer, J. Fluid Mech. 32, 173 (1968).

[39] M. Gaster, The development of a two-dimensional wavepacket in a growing boundary layer, Proc. R. Soc. Lond. 384, 317 (1982).

[40] M. Gaster, Growth of disturbances in both space and time, Phys. Fluids 11, 723 (1968).

[41] A. D. D. Craik, The development of wavepackets in unstable flows, Proc. R. Soc. Lond. 373, 457 (1981).

[42] P. Huerre and P. A. Monkewitz, Local and global instabilities in spatially developing flows, Annu. Rev. Fluid Mech. 22, 473 (1990).

[43] D. H. Bailey and P. N. Swarztrauber, A fast method for the numerical evaluation of continuous Fourier and Laplace transforms, SIAM J. Sci. Comput. 15, 1105 (1994).

[44] C. M. Bender and O. S. A., Advanced Mathematical Methods for Scientists and Engineers (McGraw-Hill, New York, 1978).

[45] R. Castrejon-Pita, A. Castrejon-Pita, S. Thete, K. Sambath, I. Hutchings, J. Hinch, J. Lister, and O. Basaran, Plethora of transitions during breakup of liquid filaments, Proc. Natl. Acad. Sci. U.S.A. 112 (2015).

[46] J. Eggers, Universal Pinching of $3 \mathrm{~d}$ Axisymmetric Free-Surface Flow, Phys. Rev. Lett. 71, 3458 (1993).

[47] S. A. Berger, Initial-value stability analysis of a liquid jet, SIAM J. Appl. Math. 48, 973 (1988).

[48] L. Rayleigh, On the instability of a cylindrical fluid surfaces, Philos. Mag. 34, 177 (1892). 